\title{
Supplementary Information \\ IMPROVING THE HETEROLOGOUS PRODUCTION OF FUNGAL PEROXYGENASES THROUGH AN EPISOMAL PICHIA PASTORIS PROMOTER AND SIGNAL PEPTIDE SHUFFLING SYSTEM
}

\author{
Pascal Püllmann\# and Martin J. Weissenborn" ${ }^{\# *}$
}

*Corresponding author. Email: martin.weissenborn@ipb-halle.de

\#Leibniz Institute of Plant Biochemistry, Weinberg 3, 06120 Halle (Saale), Germany

+Institute of Chemistry, Martin-Luther-University Halle-Wittenberg, Kurt-Mothes-Straße 2, 06120 Halle (Saale), Germany 


\section{Material and Methods}

Chemicals. Solvents were used as received without further purification. Acetone was utilised as GC ultra-grade ( $\geq 99,9 \%)$ from Carl Roth (Karlsruhe, DE). Acetonitrile was purchased from Merck (Darmstadt, DE) in gradient grade for LC ( $\geq 99,9 \%$ ). Methanol (LC-MS grade $\geq$ 99,9\%) was used from Honeywell Chemicals (Seelze, DE). 5-Nitro-1,3-benzodioxole (NBD; $98 \%$ purity) and 2,2'-Azino-bis(3-ethylbenzothiazoline-6-sulfonic acid) diammonium salt (ABTS) were retrieved from Sigma-Aldrich (Hamburg, DE). 4-Nitrophenyl laurate was purchased from TCI Chemicals (Tokyo, JP).

Enzymes and cultivation media. For cultivation of E. coli cells terrific broth (TB) media from Carl Roth (Karlsruhe, DE) was used. Peptone and Yeast extract were purchased from Formedium (Hunstanton, GB). Yeast nitrogen base (without amino acids) and Hygromycin B solution $(50 \mathrm{mg} / \mathrm{ml}$ ) were purchased from Carl Roth (Karlsruhe, DE). PNGase F and BsaI were purchased from New England Biolabs (Ipswich, US). BbsI and FastDigest AscI were purchased from ThermoFisherScientific (Waltham, US) and T4 DNA Ligase from Promega (Madison, US).

\section{General Procedures}

Expression plasmid construction (pPAP004) for Pichia pastoris. The design of the episomal backbone plasmid pPAP004 is based on a previously constructed Pichia system consisting of two episomal expression plasmids (pPAP001 and pPAP002) and an integrative plasmid (pPAP03) for subsequent stable genomic integration. ${ }^{1}$ The level 1 Golden Gate based shuttle expression plasmid pPAP004 was therefore PCR amplified (Phusion Polymerase) from pPAP001 excluding the pGAP promoter and assembled by Golden Gate cloning using BsmBI. It can be propagated in E. coli (Ampicillin resistance) as well as $P$. pastoris (Hygromycin B resistance). To enable episomal plasmid propagation in $P$. pastoris, the plasmid is equipped with a previously described functional ARS sequence, which was originally PCR amplified from Kluveromyces lactis genomic DNA. ${ }^{2,3}$

As placeholder for a target gene sequence as well as color selection marker, a lacZ cassette (approx. 600 bp) is integrated. Upon digestion with BsaI the lacZ cassette is released and a fitting open reading can be integrated in frame (e.g. promoter-Signal Peptide-Gene-C-terminal Tag) into the plasmid, thereby reconstituting a fully functional expression plasmid. For the stable integration of transcription units into the genome of $P$. pastoris, a third universal integrative plasmid (pPAP003) was utilized. This shuttle plasmid was previously constructed, which can be propagated in E. coli (Kanamycin resistance) as well as P. pastoris (Hygromycin B resistance). As placeholder for a target transcription unit a lacZ cassette is integrated, enabling blue/white selection of transformants based on the conversion of X-Gal. Upon digestion with BbsI the lacZ cassette is released and a fitting transcription unit (Promoter- ORF- Terminator) can be integrated (derived from respective pPAP004 episomal plasmids as donors) into the plasmid, thereby reconstituting a fully functional integration plasmid which can then be used for transformation.

Construction of Level 0 parts. Individual assembly parts (genes and promoters) were cloned as individual parts into the respective pAGM9121 Level 0 universal acceptor plasmid. All new gene parts (CalB, Mr2 Laccase, Mfe UPO, MhiUPO and DcaUPO) were purchased as double stranded DNA parts from Twist Bioscience (San Francisco, US), codon optimized for Pichia pastoris, bearing terminal BbsI recognition sites and pre-designed overhangs for correct assembly into pAGM9121.

In the case of the construction of the promoter library different strategies were employed. All promoter parts were cloned as individual parts into the respective pAGM9121 Level 0 and contain the same Kozak Sequence $\left({ }^{-10}\right.$ ATCATACAAA ${ }^{0}$ ATG $)$. Several promoter parts $\left(P_{P p A O X I}\right.$, $\left.P_{P p F D H 1}, P_{P p \text { DAs } 1,} P_{P p \text { DAS2 }}, P_{P p \text { ALD } 4)}\right)$ were PCR amplified and derived from a previously introduced Golden Gate based $P$. pastoris assembly system, coined GoldenPics. ${ }^{4}$ These parts are particularly suitable since they have been already domesticated regarding internal BsaI and BbsI recognition sequences. $P_{P p \text { FLD } 1}$ and $P_{P p \text { PMP20 }}$ were PCR amplified from genomic Pichia pastoris DNA. The $P_{H p M O X}$ element was amplified from genomic Hansenula polymorpha DNA in a comparable manner. Since the genomic sequences of $P_{H p \text { FMD }}$ and $P_{H p D H A S}$ contain internal recognition sites, both parts were purchased as double stranded DNA parts from Twist Bioscience (San Francisco, US) as described above and internal BsaI/BbsI recognition sites excluded by single nucleotide exchenges. All Level 0 parts were constructed by Golden Gate digestion ligation reactions (+BbsI and T4-Ligase) using pAGM9121 as acceptor plasmid. All parts were subsequently confirmed by Sanger sequencing.

Golden Gate Cloning of Expression Plasmids. The expression plasmid pPAP004 was used as respective acceptor plasmid for the assembly of the individual tetrapartite expression units ( $\mathbf{5}^{\prime}$ promoter-Signal Peptide-Gene-C-terminal Tag $\left.\mathbf{3}^{\prime}\right)$. The individual parts were thereby derived as parts from standard level 0 plasmids (pAGM9121 backbone), which can be released from the pAGM9121 backbone upon BsaI restriction digest.

Golden Gate reactions were performed in a total volume of $15 \mu \mathrm{L}$. The final reaction volume contained 1-fold concentrated T4 ligase buffer. Prepared reaction mixtures containing ligase buffer, the acceptor plasmid ( $20 \mathrm{fmol})$ and the corresponding inserts as level 0 modules (Promoter, Signal Peptide, Gene, C-terminal Tag) were added to $20 \mathrm{fmol}$ each and the overall volume adjusted to $13.5 \mu \mathrm{L}$ with ddH $\mathrm{H}_{2} \mathrm{O}$. In the case of shuffling approaches 17 different pAGM9121- Signal Peptide combinations were added in equimolar ratios (1.2 fmol each) and 11 different pAGM9121- promoter combinations in an equimolar ratio (1.9 fmol each) as well. In a final step, the corresponding enzymes were quickly added. First, a volume of $0.5 \mu \mathrm{L}$ of the restriction enzyme BsaI (10 units/ $\mu \mathrm{L})$ was added and then $1 \mu \mathrm{L}(1-3 \mathrm{units} / \mu \mathrm{L})$ of $\mathrm{T} 4$ ligase. 
Golden Gate reactions where performed using a temperature cycling program (50 to 99x passes) between $37^{\circ} \mathrm{C}(2 \mathrm{~min})$ and $16^{\circ} \mathrm{C}(5 \mathrm{~min})$ and concluded by an additional enzyme inactivation step $\left(80^{\circ} \mathrm{C} ; 20 \mathrm{~min}\right)$.

The whole Golden Gate reaction volume was used to transform $100 \mu \mathrm{L}$ of chemically competent $E$. coli DH10B cells. After heat shock transformation $\left(42^{\circ} \mathrm{C} ; 90 \mathrm{sec}\right)$ and recovery the mixture (approx. $320 \mu \mathrm{L}$ ) was split into two fractions, $50 \mu \mathrm{L}$ were plated on selective LB Agar plates $\left(50 \mu \mathrm{g} \times \mathrm{mL}^{-1} \mathrm{X}\right.$-Gal; $100 \mu \mathrm{g} \times \mathrm{mL}^{-1}$ Ampicillin; $150 \mu \mathrm{M}$ IPTG) and the remaining volume used to directly inoculate $4 \mathrm{~mL}$ TB Medium $\left(100 \mu \mathrm{g} \times \mathrm{mL}^{-1}\right.$ Ampicillin) to preserve the genetic diversity of the shuffling library. The following day the success of the Golden Gate reaction was evaluated based on the performed blue/white screening, discriminating the empty plasmid (lacZ; blue) from recombined, white colonies. In general, the described protocol for ORF assembly and promoter + signal peptide shuffling as special case led to several hundred recombinant colonies with a high proportion (>90\%) of recombined, white colonies.

In the case of single defined, "unshuffled" constructs single colonies were checked for correct insert sizes by the means of colony PCR (matching pPAP004 sequencing primer). Positively identified clones were inoculated into $4 \mathrm{~mL}$ of TB-Medium $\left(100 \mu \mathrm{g} \times \mathrm{mL}^{-1} \mathrm{Ampicillin}\right)$ and corresponding plasmid DNA prepared (NucleoSpin Plasmid Kit (Macherey-Nagel, Düren, DE)). In the case of shuffled signal peptide constructs, plasmid DNA was prepared as a library by direct inoculation of the transformation mixture into liquid culture and subsequent DNA isolation (see above).

Error prone PCR. Mutagenesis libraries of TteUPO were constructed by means of error prone PCR using the wild-type gene as amplification template. Final reaction mixtures of $50 \mu \mathrm{L}(3 \%$ (v/v) DMSO; $200 \mu \mathrm{M}$ dNTP mix; $400 \mathrm{nM}$ forward and reverse primer; $10 \mathrm{mM}$ Tris pH $8.0 ; 50 \mathrm{mM} \mathrm{KCl} ; 7 \mathrm{mM} \mathrm{MgCl}_{2}$ ) contained $5 \mathrm{ng}$ template DNA and varying manganese chloride concentrations ranging from 0.01 to $0.5 \mathrm{mM}$. Amplifications (30 cycles) were performed using in-house prepared Taq-Polymerase. Resulting amplification fragments were analysed for the occurring size by means of agarose gel electrophoresis and subsequently prepared for cloning by following the instructions of the utilised preparation kit (NucleoSpin Gel and PCR Clean-Up (Macherey-Nagel, Düren, DE).

Plasmid transformation into $\boldsymbol{P}$. pastoris. Respective single plasmids or plasmid mixtures (pPAP004 backbone) were used to transform electro competent $P$. pastoris cells (X-33 strain) by the means of electroporation. Electro competent X-33 cells were prepared according to a condensed protocol for P. pastoris. ${ }^{5}$ Cells were stored in BEDS solution (10 mM bicine-NaOH pH 8.3, $3 \%$ (v/v) ethylene glycol, $5 \%$ (v/v) DMSO and $1 \mathrm{M}$ sorbitol) as $40 \mu \mathrm{L}$ aliquots $\left(-80^{\circ} \mathrm{C}\right)$ till further use.

For the transformation of episomal plasmids $60 \mathrm{ng}$ of the circular plasmid (predilution in $\mathrm{ddH}_{2} \mathrm{O}$ to a $60 \mathrm{ng} / \mu \mathrm{L}$ stock solution) were added to one aliquot of on ice thawed competent X-33 cells. The cell-plasmid mix was then transferred to an electroporation cuvette (2 mm gap) and the cuvette cooled for 10 minutes on ice prior to the transformation. Electroporation was performed using a Micropulser Device (BioRad, Hercules, US) and using manual implemented, standardized settings (1.5 kV, 1 pulse) for all transformation setups, leading to a general pulse interval of 5.4 to $5.8 \mathrm{~ms}$. Immediately after electroporation cells were recovered in $1 \mathrm{~mL}$ of ice cold YPD-Sorbitol solution (10 g/L peptone, $5 \mathrm{~g} / \mathrm{L}$ yeast extract, $500 \mathrm{mM}$ sorbitol), transferred to a new tube and incubated for one hour under rigid shaking $\left(30{ }^{\circ} \mathrm{C}, 900 \mathrm{rpm}\right)$ in a Thermomix device (Eppendorf, Hamburg, DE). After incubation cells were precipitated by centrifugation ( $5700 \mathrm{rpm}, 5 \mathrm{~min})$. The supernatant was discarded, and the cells resuspended in $200 \mu \mathrm{L}$ of fresh YPD medium. $100 \mu \mathrm{L}$ of the suspension were then plated on selective YPD Agar plates supplemented with $150 \mu \mathrm{g} / \mathrm{mL}$ Hygromycin B. Plates were incubated at $30{ }^{\circ} \mathrm{C}$ for at least $48 \mathrm{~h}$ till clearly visible colonies appeared. In general, the described setup led to the occurrence of several hundred colonies per plate.

For the transformation of integrative plasmids (pPAP003 backbone) the setup was slightly modified since in this case linearized plasmids are used for transformation. Therefore, previously prepared circular plasmid DNA was digested with AscI (Isoschizomer: SgsI). $2.5 \mu \mathrm{g}$ of the respective plasmid DNA was mixed with $3 \mu \mathrm{L}$ of 10x fold FastDigest Buffer, the volume adjusted to $29.5 \mu \mathrm{L}$ using ddH $\mathrm{H}_{2} \mathrm{O}$ and in a last step $0.5 \mu \mathrm{L}$ of FastDigest SgsI added. Digestion was performed overnight $\left(16 \mathrm{~h}, 37^{\circ} \mathrm{C}\right)$ and terminated by an enzyme inactivation step $(20$ min, $80^{\circ} \mathrm{C}$ ). Linearized plasmid DNA was then subsequently prepared according to the manufacturer instruction using a Nucleospin ${ }^{\circledR}$ Gel and PCR clean up Kit (Macherey-Nagel, Düren, DE). The transformation of $P$. pastoris was performed in a congruent manner as described before, except for using $100 \mathrm{ng}$ linearized plasmid for transformation, since the overall transformation efficiency is substantially reduced in comparison to the transformation of circular plasmid.

Plasmid transformation into $\boldsymbol{H}$. polymorpha. Respective single plasmids (pPAP004 backbone) were used to transform electro-competent H. polymorpha cells (X-33 strain) by the means of electroporation. Cells were prepared and transformed according to a previously published protocol. ${ }^{6}$ After transformation the whole volume was plated on selective YPD Agar plates supplemented with $150 \mu \mathrm{g} / \mathrm{mL}$ Hygromycin B. Plates were incubated at $37^{\circ} \mathrm{C}$ for at least $48 \mathrm{~h}$ till clearly visible colonies appeared.

Microtiter Plate cultivation expression of $\boldsymbol{P}$. pastoris and $\boldsymbol{H}$. polymorpha. For enzyme production in microtiter plate format specialized 96 half deep well plates were utilized. The model type CR1496c was purchased from EnzyScreen (Heemstede, NL) and plates were covered with fitting CR1396b Sandwich cover for cultivation. Plates and covers were flushed before every experiment thoroughly with $70 \%$ ethanol and air dried under a sterile bench until usage. Each cavity was filled with $220 \mu \mathrm{L}$ of buffered complex medium (BM) and inoculated with single, clearly separated yeast colonies using sterile toothpicks. Basic BM (20 g/L peptone; $10 \mathrm{~g} / \mathrm{L}$ yeast extract; $100 \mathrm{mM}$ potassium phosphate buffer $\mathrm{pH} 6.0 ; 1 \mathrm{x}$ YNB (3.4 g/L yeast nitrogen base without amino acids; $100 \mathrm{~g} / \mathrm{L}$ ammonium sulfate); $400 \mu \mathrm{g} / \mathrm{L}$ biotin; $3.2 \mathrm{mM}$ magnesium sulfate; $25 \mathrm{mg} / \mathrm{L}$ chloramphenicol; $50 \mathrm{mg} / \mathrm{L}$ hemoglobin; $150 \mathrm{mg} / \mathrm{L}$ Hygromycin B) was freshly prepared out of sterile stock solutions immediately before each experiment, mixed and added to the cavities.

Depending on the type of experiment different carbon source feeding strategies were employed. Therefore, defined amounts $(0.3 \% ; 0.5 \%$; $1.0 \%$ or $1.5 \%$ final) of the primary carbon sources glucose and glycerol were added to the cultivation media derived from defined stock solutions. Pure methanol was added to a final concentration of 1.5 or $2 \%(\mathrm{v} / \mathrm{v})$.

Regarding the production of $\mathrm{Mr} 2$ laccase and $\mathrm{CalB}$ some alterations regarding the cultivation media were made. CalB expression plates were prepared without the supplementation of magnesium sulphate and hemoglobin. In the case of Mr2 Laccase expression plates $3.2 \mathrm{mM}$ magnesium sulfate were replaced by $1 \mathrm{mM}$ copper sulfate and no hemoglobin added. Volume differences were adjusted with ddH $\mathrm{H}_{2} \mathrm{O}$ and the overall procedure not changed. In the case of rescreen setups of the novel peroxygenases (MhiUPO, MfeUPO and DcaUPO) medium was prepared without the supplementation of hemoglobin to avoid interference (background activity) within the utilized screening assay. 
After inoculation of the wells the plates were covered, mounted on CR1800 cover clamps (EnzyScreen) and incubated in a Minitron shaking incubator (Infors, Bottmingen, SUI) for $72 \mathrm{~h}\left(30^{\circ} \mathrm{C} ; 230 \mathrm{rpm}\right)$. After cultivation the cells were separated from the enzyme containing supernatant by centrifugation $\left(3400 \mathrm{rpm} ; 50 \mathrm{~min} ; 4^{\circ} \mathrm{C}\right)$.

Shake flask cultivation $\boldsymbol{P}$. pastoris. For large scale protein production using shake flasks genomically integrated single constructs (pPAP 003 backbone; integration into chromosomal 3' region of $P$. pastoris AOX1 gene) were chosen. These constructs were previously identified by screening at least 7 different colonies per individual construct (promoter - signal peptide - gene combination) within a MTP screening setup and choosing a respective production strain based on a high as possible, clearly distinguishable NBD, ABTS or 4-nitrophenyl laurate conversion in comparison to the background control (pPAP003 empty plasmid).

Precultures were prepared in $50 \mathrm{~mL}$ YPD medium (+ $25 \mathrm{mg} / \mathrm{L}$ chloramphenicol) and cultivated for $48 \mathrm{~h}\left(30{ }^{\circ} \mathrm{C} ; 160 \mathrm{rpm} ; 80 \%\right.$ humidity), typically resulting in a final $\mathrm{OD}_{600 \mathrm{~nm}}$ of approx. 17 to 19 . The main expression culture was inoculated with a starting optical density of 0.3 . For large scale production BM based expression media $(20 \mathrm{~g} / \mathrm{L}$ peptone; $10 \mathrm{~g} / \mathrm{L}$ yeast extract; $100 \mathrm{mM}$ potassium phosphate buffer $\mathrm{pH}$ 6.0; 1x YNB (3.4 g/L yeast nitrogen base without amino acids; $10 \mathrm{~g} / \mathrm{L}$ ammonium sulfate); $400 \mu \mathrm{g} / \mathrm{L}$ biotin; $3.2 \mathrm{mM}$ magnesium sulfate (peroxygenases) or $1 \mathrm{mM}$ copper sulfate (Mr2 Laccase); $25 \mathrm{mg} / \mathrm{L}$ chloramphenicol) was utilized. Cultivation was performed in 2,5 L Ultra yield flasks (Thomson Instrument, Oceanside, US) in a final culture volume of $500 \mathrm{~mL}$ per flask after sealing the flask with breathable Aeraseal tape (Sigma Aldrich, Hamburg, DE) allowing for gas exchange. The main cultures were incubated for further $72 \mathrm{~h}\left(25^{\circ} \mathrm{C} ; 110 \mathrm{rpm} ; 80 \%\right.$ humidity).

In the case of derepressed production $1 \%$ (w/v) Glycerol were added as carbon source for Pichia growth. In the case of the methanol induced production a two-phase feeding was applied, firstly inoculating the cells into BM medium (see above) supplemented with $0.4 \%$ (w/v) glycerol as carbon source. $24 \mathrm{~h}$ and $48 \mathrm{~h}$ after inoculation $1.0 \%(\mathrm{v} / \mathrm{v})$ of methanol were added as inducer of the respective MUT promoter. After cultivation the cells were separated from the enzyme containing supernatant by centrifugation $\left(4300 \mathrm{rpm} ; 35 \mathrm{~min} ; 4{ }^{\circ} \mathrm{C}\right)$.

Supernatant ultrafiltration and protein purification. The previously prepared supernatant was concentrated approx. 20 -fold by the means of ultrafiltration. Therefore, a Sartocon Slice 200 membrane holder (Sartorius, Göttingen, DE) was equipped with a Sartocon Slice 200 ECO Hydrosart Membrane (10 kDa nominal cut-off; Sartorius) within a self-made flow setup. The flow system for ultrafiltration was operated by an EasyLoad peristaltic pump (VWR International, Darmstadt, DE).

In a first step the cleared supernatant $(1 \mathrm{~L})$ was concentrated approx. 10 -fold to a volume of $100 \mathrm{~mL}$ and $900 \mathrm{~mL}$ of purification binding buffer $(100 \mathrm{mM}$ Tris- $\mathrm{HCl} \mathrm{pH} 8.0,150 \mathrm{mM} \mathrm{NaCl})$ were added as a buffer exchange step. This sample was then concentrated approx. 20-fold to achieve a final volume of $50 \mathrm{~mL}$.

Protein purification was implemented utilizing the C-terminal attached double Strep II Tag (WSHPQFEK), coined TwinStrep® (Iba Lifesciences, Göttingen, DE). As column material Strep-Tactin®XT Superflow ${ }^{\circledR}$ columns ( $1 \mathrm{~mL}$ or $5 \mathrm{~mL}$; Iba Lifesciences) were chosen and the flow system operated by an EasyLoad peristaltic pump (VWR). In a first step the column was equilibrated with 5 column volumes (CVs) binding buffer. The concentrated sample $(50 \mathrm{~mL})$ was filter sterilized $(0.2 \mu \mathrm{m}$ syringe filter $)$ and applied to the column with an approximate flow rate of $1 \mathrm{~mL} / \mathrm{min}$. After application the column was washed with $6 \mathrm{CVs}$ binding buffer. Elution was performed based on binding competition with biotin, therefore approx. $2 \mathrm{CV}$ of elution buffer $(100 \mathrm{mM}$ Tris- $\mathrm{HCl} \mathrm{pH} 8.0,150 \mathrm{mM} \mathrm{NaCl} ; 50 \mathrm{mM}$ biotin) were applied to the column. The pooled elution fraction was then dialysed overnight $\left(4^{\circ} \mathrm{C}\right)$ against $5 \mathrm{~L}$ of storage buffer $(100 \mathrm{mM}$ potassium phosphate $\mathrm{pH}$ 7.0) using ZelluTrans dialysis tubing (6-8 kDa nominal cut-off; Carl Roth) and the recovered, dialyzed sample stored at $4{ }^{\circ} \mathrm{C}$ till further use.

Purification of DcaUPO by hydrophobic interaction chromatography (HIC). The enzyme DcaUPO was purified by the means of hydrophobic interaction chromatography (HIC). Therefore, the cultivation supernatant was first concentrated as described above to a volume of approx. $100 \mathrm{~mL}$ and approx. $950 \mathrm{~mL}$ of equilibration buffer $(50 \mathrm{mM}$ potassium phosphate; $\mathrm{pH} 7.0)$ added. The sample was concentrated once again to a final volume of $50 \mathrm{~mL}$ and filter sterilized $(0.2 \mu \mathrm{m}$ syringe filter). Afterwards a concentrated ammonium sulfate solution (3.5 $\mathrm{M}$ stock solution) was slowly added using a syringe pump for dosing till reaching a final concentration of $1 \mathrm{M}$ within the sample after 40 min under stirring. Precipitated proteins were separated by centrifugation $\left(12000 \mathrm{rpm} ; 15 \mathrm{~min} ; 20^{\circ} \mathrm{C}\right)$ from the soluble fraction. A $1 \mathrm{~mL}$ HiTrap $^{\text {TM }}$ Octyl FF column was used as material (GE Healthcare, Uppsala, SWE) and equilibrated with binding buffer (33 mM potassium phosphate $\mathrm{pH} 7.0 ; 1 \mathrm{M} \mathrm{NH}_{4} \mathrm{SO}_{4}$ ). The sample was applied to the column with an approximate flow rate of $1 \mathrm{~mL} / \mathrm{min}$ and the column washed afterwards with approx. $20 \mathrm{CV}$ of binding buffer. Primary elution was achieved with a sharp step elution by switching to low salt elution buffer (33 mM potassium phosphate $\mathrm{pH} 7.0)$ and approx. $4 \mathrm{CV}$ of elution fraction retrieved. A tightly bound fraction of $D c a \mathrm{UPO}$ remained on the column and was then eluted as highly pure enzyme using pure ethylene glycol as eluent. The ethylene glycol elution fraction was then dialyzed overnight $\left(4^{\circ} \mathrm{C}\right)$ against $5 \mathrm{~L}$ of storage buffer $(100 \mathrm{mM}$ potassium phosphate $\mathrm{pH} 7.0)$ using ZelluTrans dialysis tubing (6-8 $\mathrm{kDa}$ nominal cut-off; Carl Roth) and the recovered, dialyzed sample stored at $4{ }^{\circ} \mathrm{C}$ till further use.

Plasmid preparation of episomal plasmids from yeast. Yeast plasmids of identified clones were recovered by the means of digestive Zymolase cell treatment and alkaline cell lysis. Therefore streak-outs of previously identified suitable promoter-signal peptide combinations on YPD-Agar plates were used. A small amount of cells was collected with a sterile toothpick and resuspended in 1 mL of washing buffer $(10 \mathrm{mM}$ EDTA NaOH; pH 8.0) by light vortexing. Cells were subsequently pelleted $(5000 \times \mathrm{g} ; 10$ min $)$ and the supernatant discarded. Afterwards cells were resuspended by light vortexing in $600 \mu \mathrm{L}$ of Sorbitol Buffer $(1.2 \mathrm{M}$ sorbitol, $10 \mathrm{mM} \mathrm{CaCl} 2,100 \mathrm{mM} \mathrm{Tris-HCl} \mathrm{pH} 7.5$, $35 \mathrm{mM} \beta$-mercaptoethanol) and 100 units of Zymolase (Sigma Aldrich, Hamburg, DE) added followed by an incubation step for 120 min $\left(30{ }^{\circ} \mathrm{C} ; 650 \mathrm{rpm}\right)$ to facilitate yeast cell wall digestion. After incubation cells were pelleted by centrifugation $(2000 \times \mathrm{g} ; 10 \mathrm{~min})$ the supernatant discarded, and the plasmid preparation started with an alkaline lysis step following the manufacturer's instructions (NucleoSpin Plasmid Kit, Macherey Nagel). In a final step the yeast derived episomal plasmids were eluted in $25 \mu \mathrm{L}$ elution buffer (5 mM Tris-HCl pH 8.5 ) and the whole eluate used to transform one aliquot of E. coli DH10B (transformation as described above), plating the whole transformation mix on a selective LB-Agar plate $\left(50 \mu \mathrm{g} \times \mathrm{mL}^{-1} \mathrm{X}\right.$-Gal; $100 \mu \mathrm{g} \times \mathrm{mL}^{-1}$ Ampicillin; $150 \mu \mathrm{M}$ IPTG).

On the following day single colonies were picked, inoculated into $4 \mathrm{~mL}$ of TB medium $\left(100 \mu \mathrm{g} \times \mathrm{mL}^{-1}\right.$ Ampicillin $)$, plasmid prepared (NucleoSpin Plasmid Kit) and sent for Sanger Sequencing to elucidate the respective sequence of the open reading frame (Eurofins Genomics). 
Protein concentration determination and purification yield. Protein concentrations of the respective protein samples were determined after dialysis of the elution fractions (storage buffer: $100 \mathrm{mM}$ potassium phosphate $\mathrm{pH}$ 7.0). In this regard the colorimetric BCA assay was utilized, employing a Pierce ${ }^{\mathrm{TM}}$ BCA Protein Assay Kit (ThermoFisherScientific, Waltham, US) following the instructions of the manufacturer. Samples were measured in biological triplicates $(25 \mu \mathrm{L}$ of a previously diluted sample) and concentrations calculated based on a previously performed calibration curve using BSA $(0-1000 \mu \mathrm{g} / \mathrm{mL})$ as reference protein.

To determine the overall yield of enzyme production per litre of culture volume the determined concentration in the elution fraction was extrapolated to the overall NBD/ABTS/4-Nitrophenyl laurate activity of the sample after ultrafiltration (column load). This calculation is based on the fact that all substrates are suitable for specific activity measurement, since negligible background signals of empty plasmid control (pPAP003 and pPAP004) regarding the substrate's conversions were observed.

Therefore, samples of every purification step (load, flow-through, wash and elution fraction) were collected and NBD/ABTS/4-Nitrophenyl laurate conversion rates of the respective fractions measured immediately after purification by testing of suitably diluted samples. In the case of non-complete binding of the enzyme fraction to the affinity column (remaining enzymatic activity in flow-through fractions) this remaining non-bound enzyme amount was taken into consideration for calculation for the overall volumetric production yield per litre. Therefore, the via BCA assay determined protein concentration of the elution fraction was extrapolated to the activity of the respective non-bond fraction, assuming a comparable specific enzyme activity for NBD/ABTS/4-Nitrophenyl laurate conversion and considering the volumes of the respective fractions, leading to an approximate enzyme titre per litre of shake flask culture.

Resting-state absorption and heme CO complex measurements. The pooled and dialyzed elution fractions (100 mM potassium phosphate $\mathrm{pH}$ 7.0) were used to record absorption spectra of the respective enzymes in their native, resting state (ferric iron; Fe ${ }^{3+}$ ). For all measurements a QS High precision Quartz Cell cuvette (Hellma Analytics, Müllheim, DE) with a path length of 10 mm was used. Spectra were recorded on a Biospectrometer Basic device (Eppendorf, Hamburg, DE) in the spectral range from 250 to $600 \mathrm{~nm}$ (interval: $1 \mathrm{~nm}$ ) and subtracting the utilized storage buffer (100 $\mathrm{mM}$ potassium phosphate $\mathrm{pH}$ 7.0) as previous blank measurement.

Heme carbon dioxide spectra (CO assay) were recorded after reducing the heme iron to its ferrous form (Fe ${ }^{2+}$ ). Therefore, a spatula tip of sodium dithionite as reducing agent was added to $1 \mathrm{~mL}$ of a respective enzyme fraction (see above) and mixed thoroughly till complete dissolution. This sample was split into two fractions and one fraction immediately flushed with a constant carbon monoxide flow for 2 min (approx. 1 bubble/sec) to obtain the thiolate-heme carbon monoxide complex. The sample was immediately transferred to a cuvette and absorption measured as described above, subtracting the reduced non-treated sample as blank measurement.

In case of $M f e \mathrm{UPO}$ and $M h i \mathrm{UPO} \mathrm{CO}$-Assay was also used to quantify volumetric yields of UPO production in comparison with $M t h \mathrm{UPO}$. Therefore, a spatula tip of sodium dithionite as reducing agent was added to $1 \mathrm{~mL}$ of the enriched (approx. 10x fold) respective enzyme fraction (see above) after ultrafiltration (in $100 \mathrm{mM}$ potassium phosphate $\mathrm{pH}$ 6.0) and mixed thoroughly till complete dissolution. This sample was split into two fractions and one fraction immediately flushed with a constant carbon monoxide flow for 2 min (approx. 1 bubble/sec) to obtain the thiolate-heme carbon dioxide complex. The sample was immediately transferred to a cuvette and absorption measured as described above, subtracting the reduced non-treated sample as blank measurement. pPAP003 was measured as a negative control, exhibiting no detectable maximum around $450 \mathrm{~nm}$. Due to the high sequence homology to $M t h \mathrm{UPO}(>90 \%)$ the volumetric yield of $M t h \mathrm{UPO}(22.4 \mathrm{mg} / \mathrm{L})$ was set as $100 \%$ and both enzyme yields (MfeUPO and MhiUPO) calculated based on their maximal peak intensity when compared to the peak intensity of a MthUPO sample which was prepared under identical conditions.

SDS Gel analysis and PNGaseF treatment. Obtained elution fractions of the respective enzymes were analyzed for the apparent molecular weight and overall purity after the performed one step TwinStrep purification by the means of SDS PAGE. Therefore, samples of the column load (after ultrafiltration; see above), elution fractions after dialysis and deglycosylated elution fraction samples were analyzed on self-casted SDS PAGE (10 or $12 \%$ of acrylamide) utilizing a Bio-Rad (Hercules, US) Mini-Protean ${ }^{\circledR}$ Gel electrophoresis System. For the purpose of molecular weight determination, a defined PageRuler Prestained Protein Ladder (ThermoFisherScientific, Waltham, US) was included, covering a MW range between 10 and $180 \mathrm{kDa}$. Proteins were visualized using a colloidal Coomassie G-250 staining solution.

To obtain N-type deglycosylated protein samples elution fractions were enzymatically treated with Peptide-N-Glycosidase F (PNGaseF) from Flavobacterium meningosepticum, which is capable of cleaving Asparagine linked high mannose type glycan structures as typically occurring in P. pastoris derived glycosylation patterns. Therefore, $45 \mu \mathrm{L}$ of a respective elution fraction were mixed with $5 \mu \mathrm{L}$ of denaturing Buffer (final $0.5 \%$ SDS; $40 \mathrm{mM}$ DTT) and denatured for 10 minutes $\left(100{ }^{\circ} \mathrm{C}\right)$. After a cooling step to room temperature $6 \mu \mathrm{L}$ of NP-40 solution (final: $1 \%$ ) and $6 \mu \mathrm{L}$ of GlycoBuffer2 (500 mM sodium phosphate; $\mathrm{pH} 7.5)$ were added and the solution thoroughly mixed. Finally, $1 \mu \mathrm{L}$ of PNGaseF (New England Biolabs, Ipswich, US) was added and the sample incubated under light shaking ( $37^{\circ} \mathrm{C}$ ) for 3 hours. After incubation the sample was prepared for further analysis by adding $5 \mathrm{x}$ fold SDS sample buffer and subsequent SDS PAGE analysis executed as described before. 


\section{Supplemental Figure 1 General design principle of Golden Gate Assembly}

Schematic overview of designed Golden Gate circuit. (1) Individual modules (promoter, signal peptide, UPO gene,C terminal Tag) are cloned as Level 0 modules into pAGM9121 and flanked by BsaI recognition sites. Upon BsaI digest parts are released bearing the indicated $4 \mathrm{bp}$ overhangs for subsequent reassembly. (2) The tetrapartite assembly can be correctly assembled into pPAP004 (BsaI digest). (3) Whole transcription units (promoter-ORF-terminator) can be swapped into a genomic integration plasmid (BbsI digest).

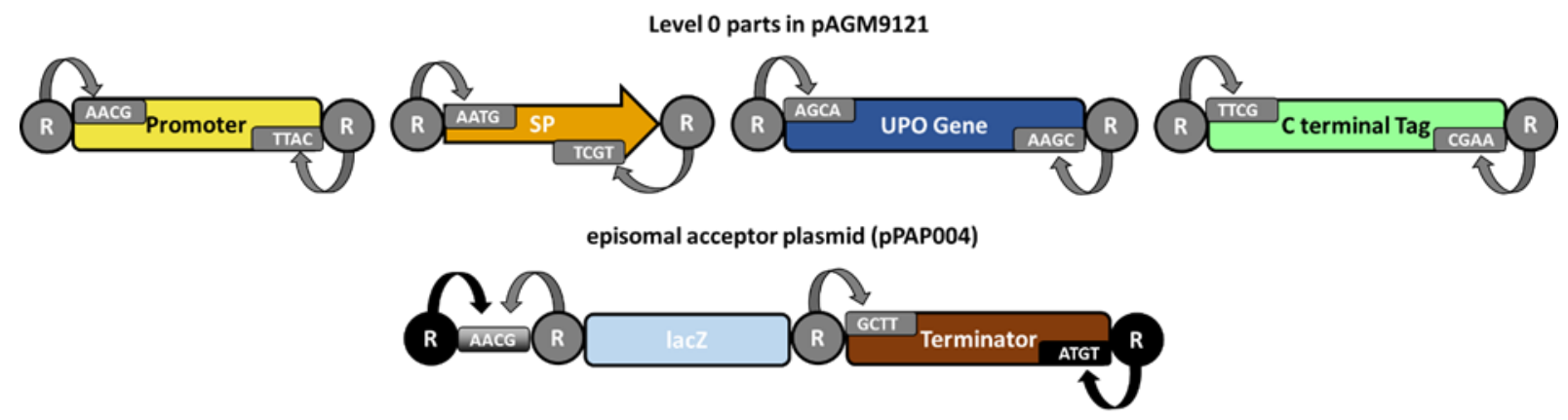

genomic integration plasmid (pPAP003)

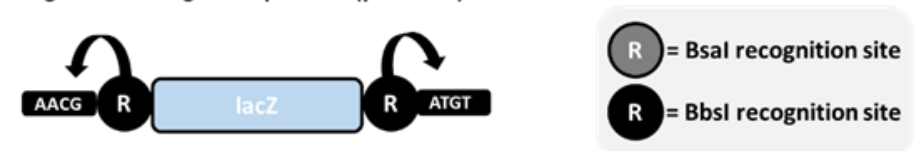

\section{Supplemental Figure 2 Experimental workflow of the two-layered Pichia pastoris system}

Schematic overview of the designed episomal and integrative circuit with indication of the minimal time requirements for the respective procedures.

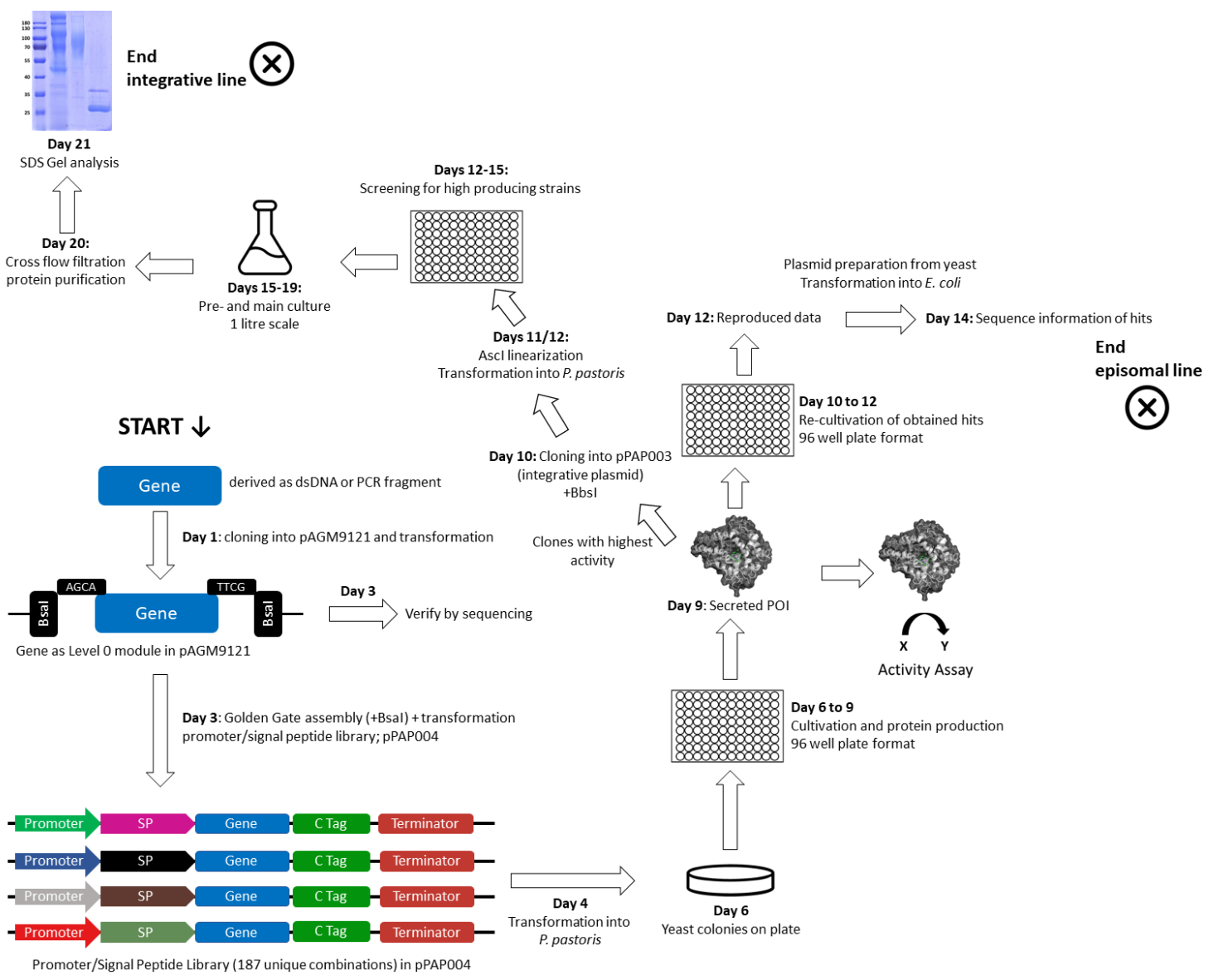




\section{Supplemental Figure 3 Time course of derepression screening TteUPO constructs}

Respective constructs (Px-Sce-Invertase 2 SP-TteUPO and PHpFMD- Ani- $\alpha$ Amylase SP-TteUPO) were cultivated in 96 well plates for $100 \mathrm{~h}$ and individual samples retrieved at the indicated time points. For each time point two wells of the respective construct were unified, cells spun down and UPO activity in the supernatant $(40 \mu \mathrm{L}$ volume $)$ determined by the means of NBD conversion measurements.
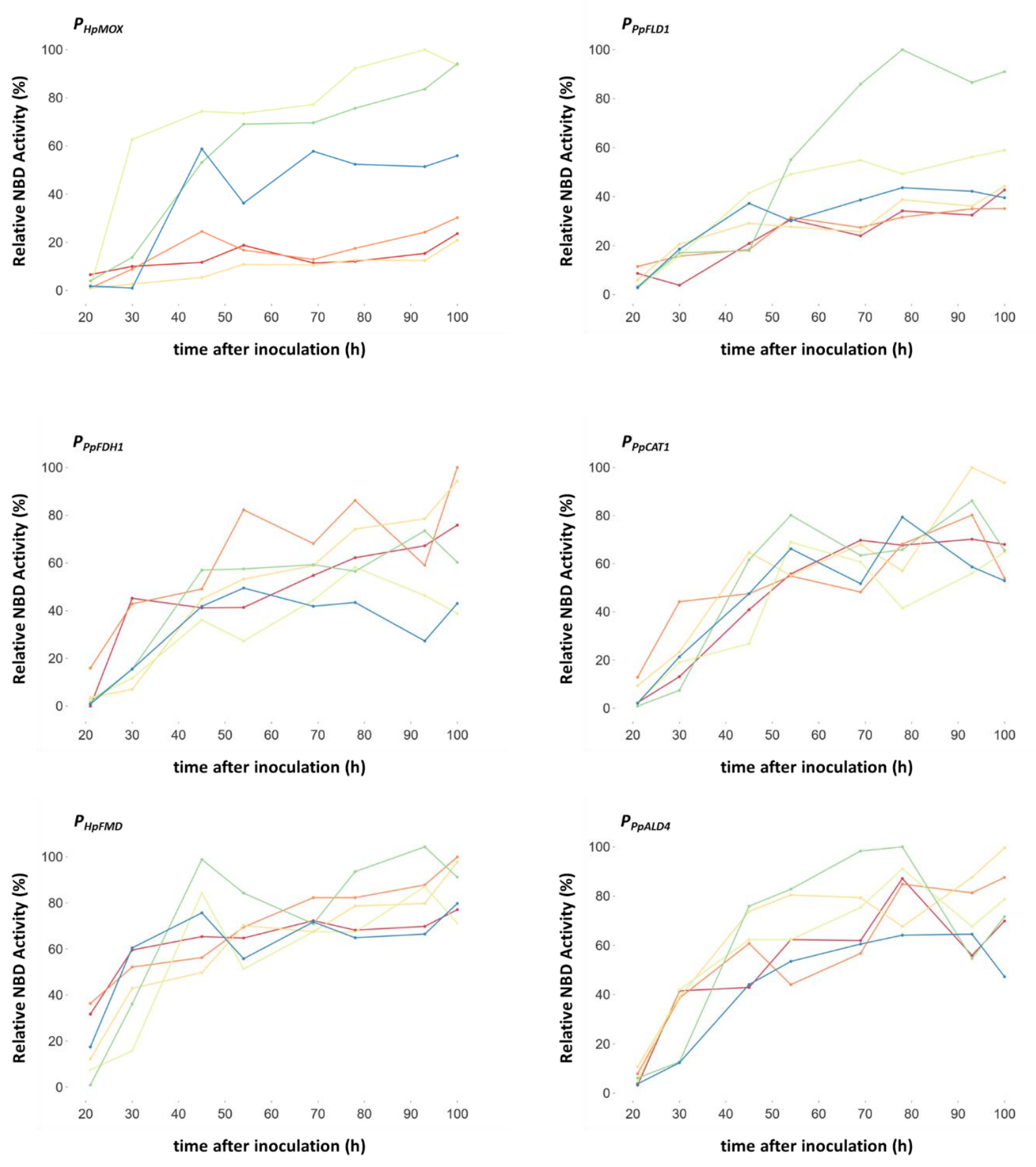

$0.5 \%$ Glycerol

$1.0 \%$ Glycerol

$1.5 \%$ Glycerol

$0.5 \%$ Glycerol; $1.5 \%$ Methanol

$1.0 \%$ Glycerol; $1.5 \%$ Methanol

$1.5 \%$ Methanol; $1.5 \%$ Methanol 


\section{Supplemental Figure 4 Testing synergistic effects of promoter and signal peptide usage}

Constructs of TteUPO and the respective promoter/signal peptides were cultivated for $72 \mathrm{~h}$ (6 biological replicates) and UPO activity measured in $20 \mu \mathrm{L}$ of supernatant. The highest mean activity was set as $100 \%$ and all values normalized accordingly.

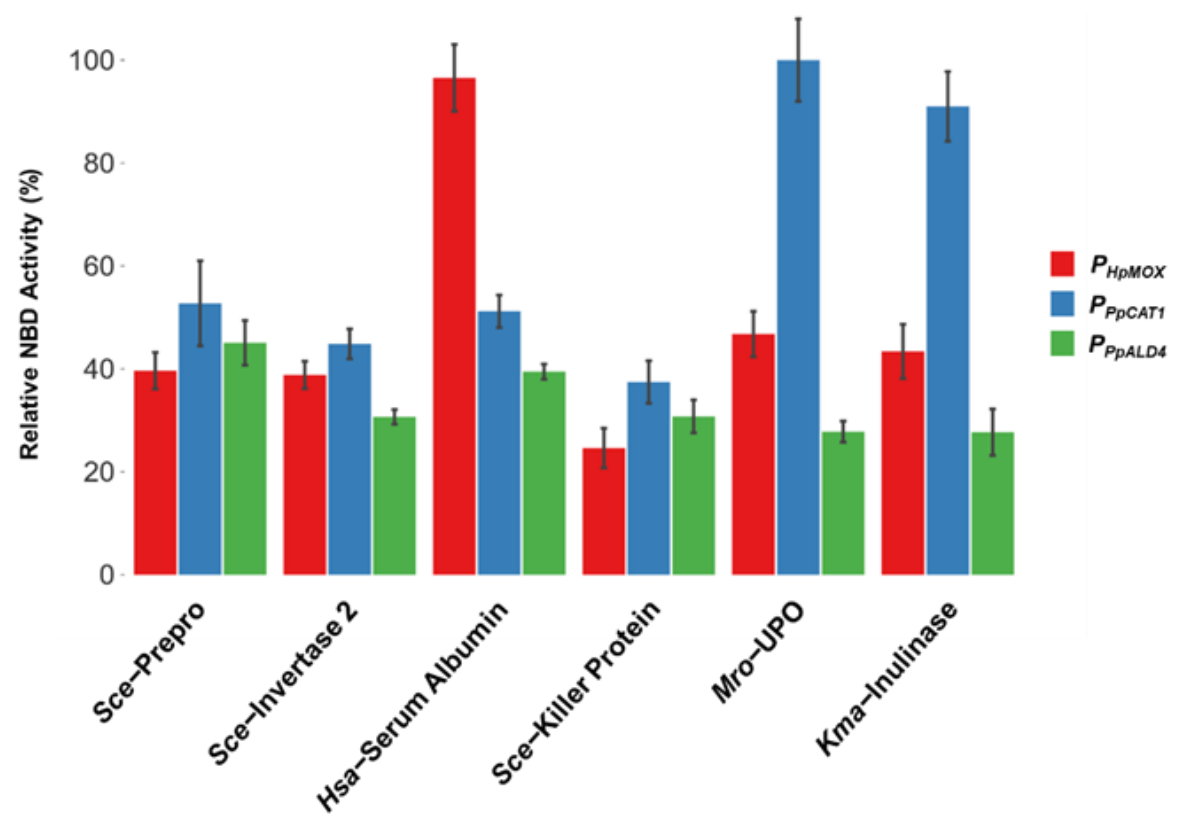

\section{Supplemental Figure 5 Screening landscape TteUPO}

Primary screening results for the constructed promoter signal peptide shuffling library in combination with TteUPO. 384 colonies (4 MTPs; 8 positive and negative controls respectively) were tested for NBD conversion. All clones have been ranked by their relative activity towards the substrate NBD. Included positive controls ( $P$ Pp CAT1-Mro-UPO) are indicated with a (+), the empty plasmid backbone (pPAP004) was used as negative control (-). $5 \%$ of the activity of the positive controls was chosen as threshold for clones to be classified as active (dashed line). Out of all tested clones, in total $242(66 \%)$ exhibited clear activity towards NBD.

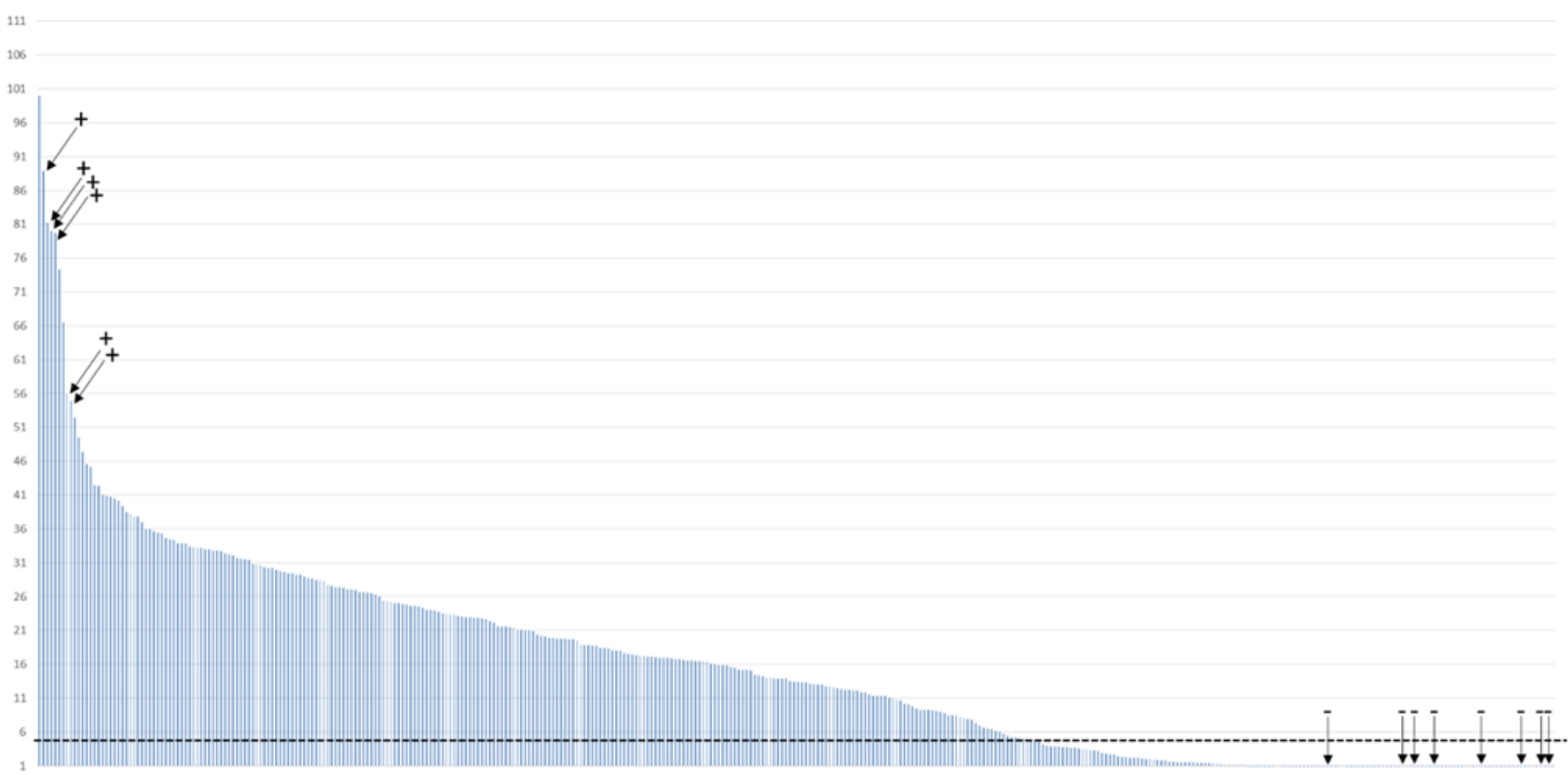




\section{Supplemental Figure 6 Screening landscape MthUPO}

Primary screening results for the constructed promoter signal peptide shuffling library in combination with MthUPO. 384 colonies (4 MTPs; 8 positive and negative controls respectively) were tested for NBD conversion. All clones have been ranked by their relative activity towards the substrate NBD. Included positive controls ( $P_{P p \text { FLD1- }}-S_{c e}-\alpha$ Galactosidase) are indicated with a (+), the empty plasmid backbone (pPAP004) was used as negative control (-). $10 \%$ of the activity of the positive controls was chosen as threshold for clones to be classified as active (dashed line). Out of all tested clones, in total 205 (56\%) exhibited clear activity towards NBD.

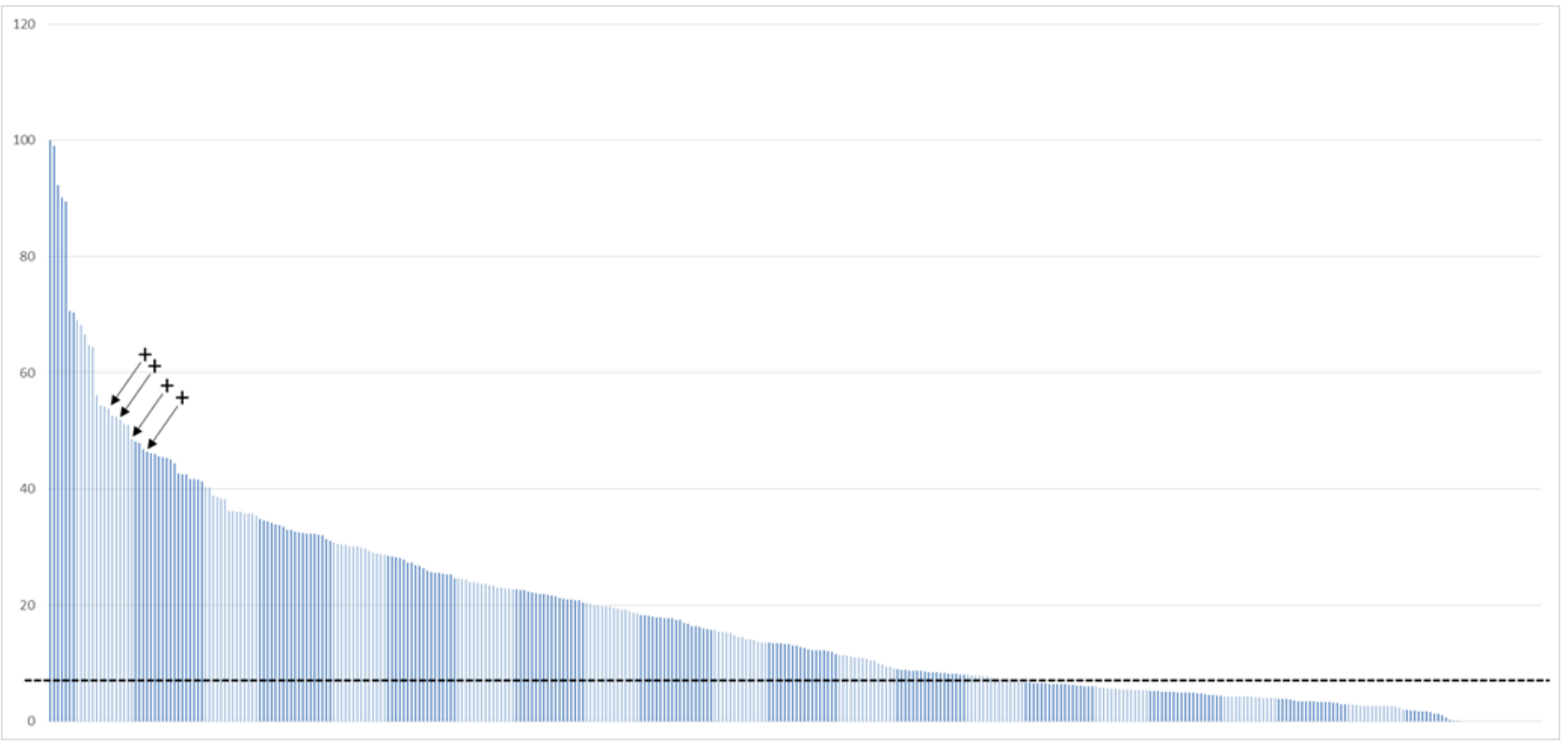

\section{Supplemental Figure 7 Screening landscape AaeUPO*}

Primary screening results for the constructed promoter signal peptide shuffling library in combination with AaeUPO*. 384 colonies (4 MTPs; 8 negative controls respectively) were tested for NBD conversion. All clones have been ranked by their relative activity towards the substrate NBD. Included positive controls ( $P_{P p \mathrm{CAT} 1^{-}}$Gma-UPO) are indicated with a $(+)$, the empty plasmid backbone (pPAP004) was used as negative control (-). $20 \%$ of the activity of the positive controls was chosen as threshold for clones to be classified as active (dashed line). Out of all tested clones, in total 28 (7\%) exhibited clear activity towards NBD.

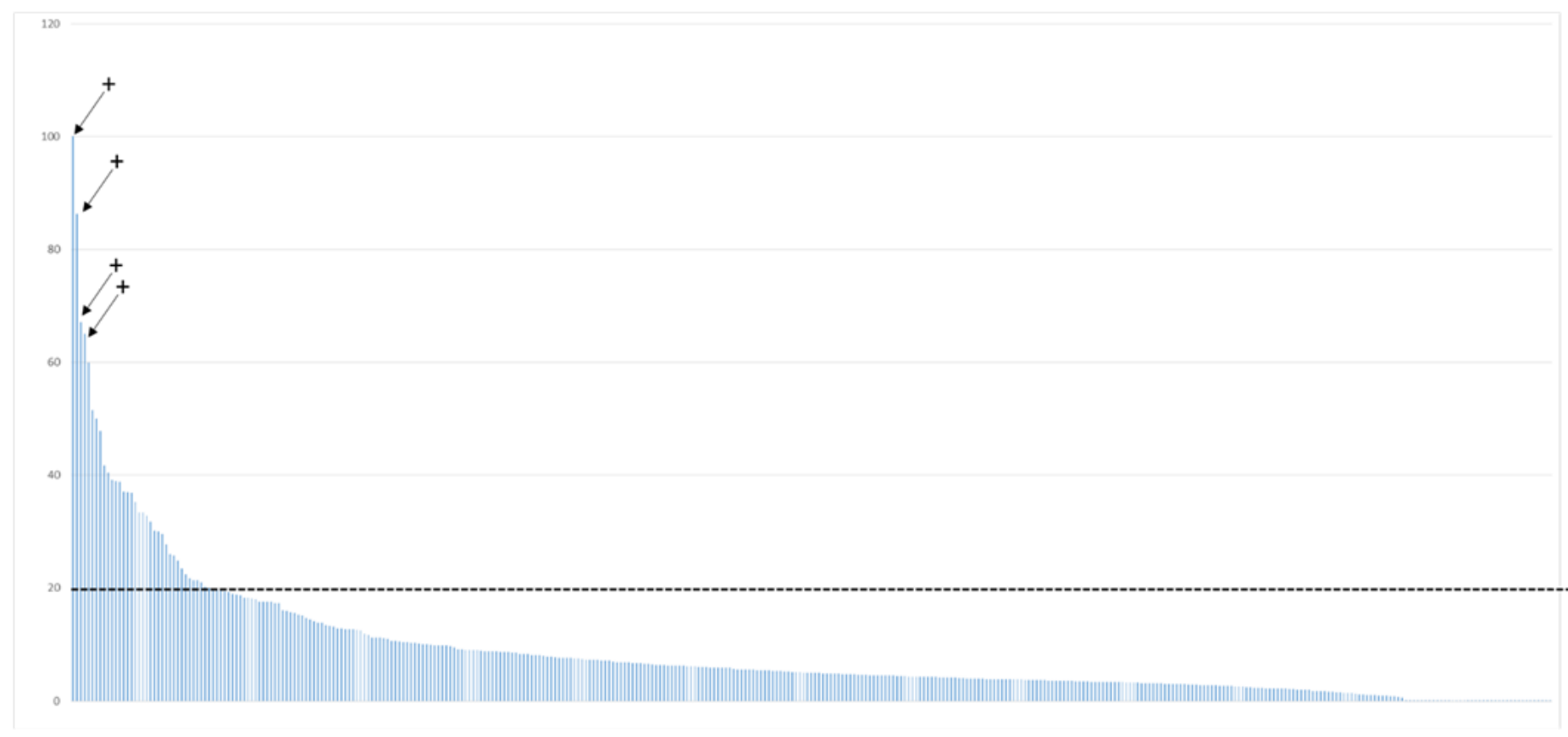


Supplemental Figure 8 Activity distribution of carbon source screening episomal/integrative constructs TteUPO, MthUPO, AaeUPO*

6 biological replicates of the respective episomal and integrative constructs were tested towards NBD conversion. The highest sample mean within one group (out of the 12 tested conditions) was set as $100 \%$ activity and all conversion values normalized accordingly.

\section{Scheme of Boxplots:}

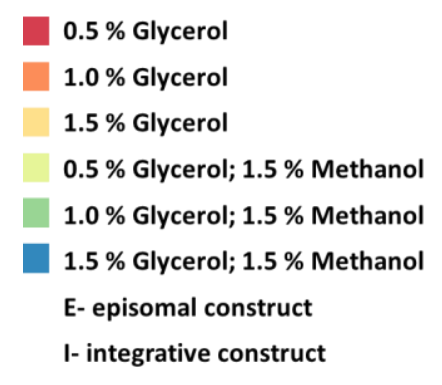

\section{TteUPO}

$P_{\text {HPFMD }}$ Ani- $\alpha$ Amylase

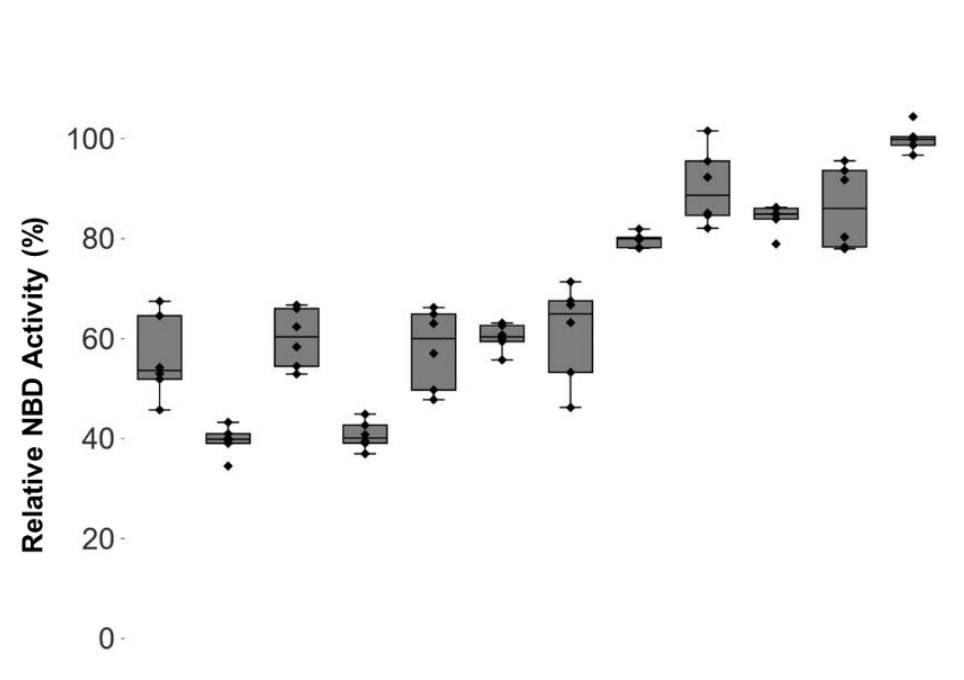

PPDCAT1- MrO-UPO

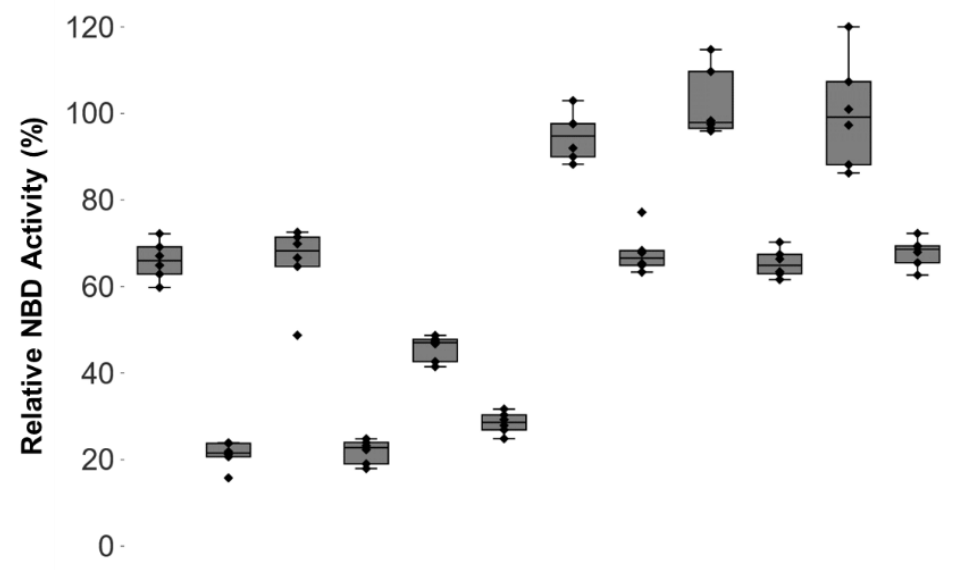




\section{MthUPO}

PHPFMD- Gga-Lysozym

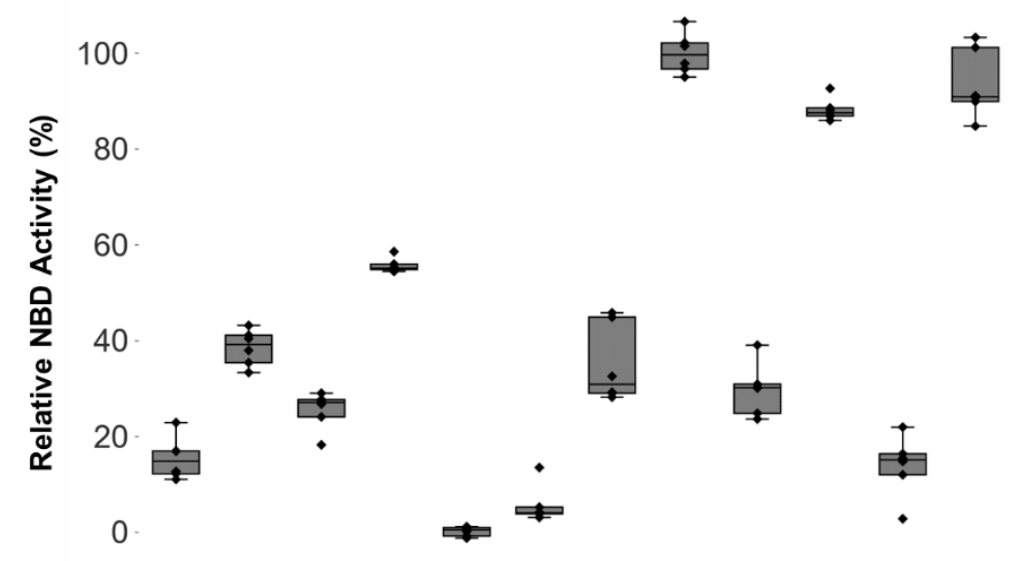

$P_{P p F L 1^{-}}$Hsa-Serum Albumin

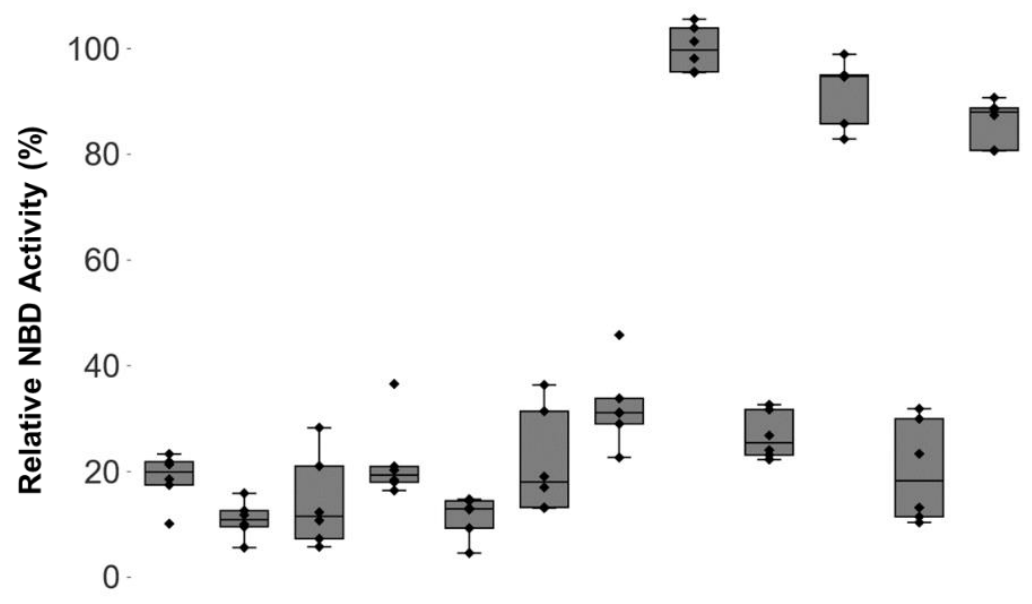


AaeUPO*

PHPFMD- Gma-UPO

$P_{\text {HрMох }}$ Gma-UPO
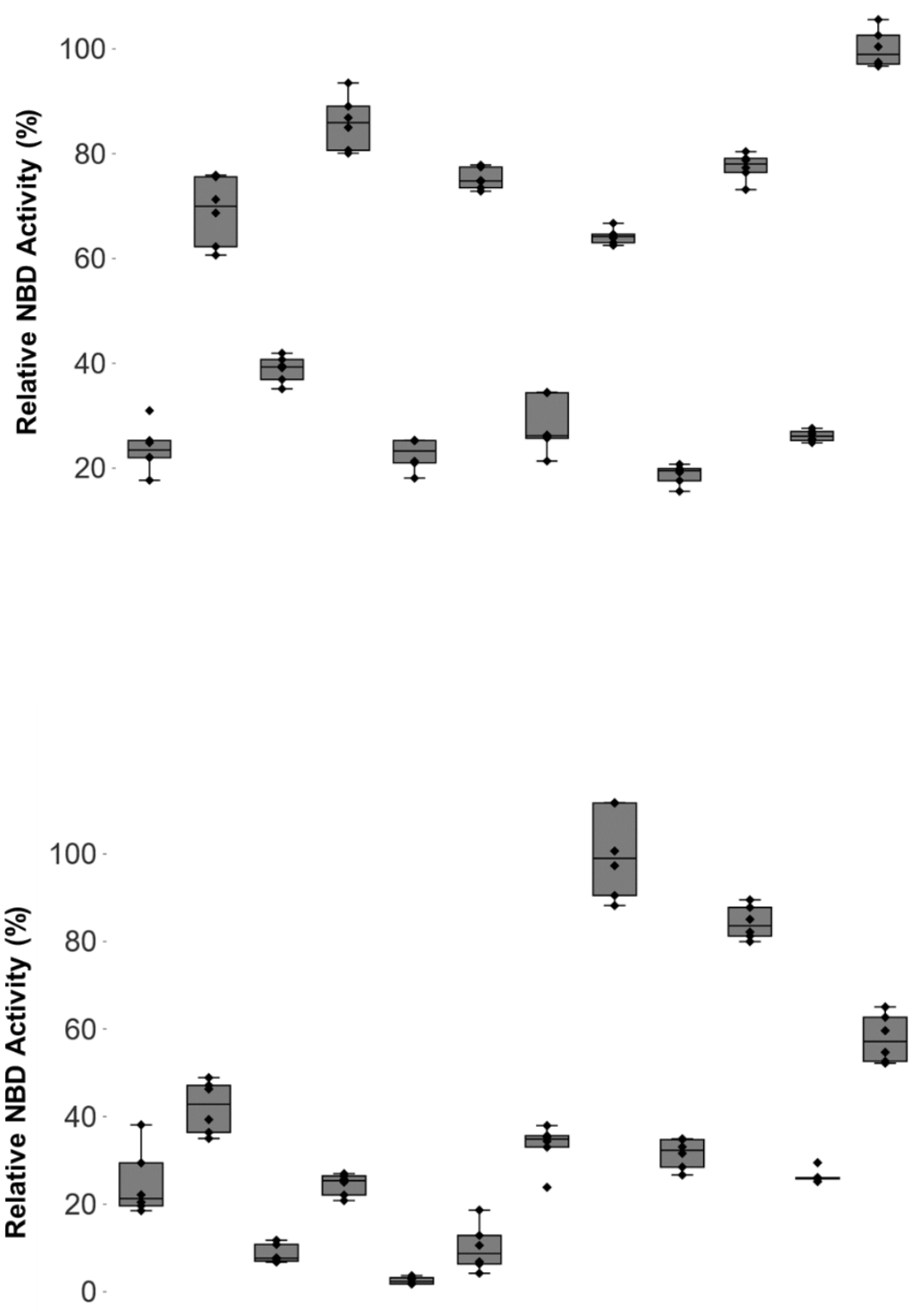


\section{Supplemental Figure 9 Screening landscape DcaUPO}

Primary screening results for the constructed promoter signal peptide shuffling library in combination with DcaUPO. 368 colonies (4 MTPs; 8 positive and negative controls respectively) were tested for DMP conversion. All clones have been ranked by their relative activity towards the substrate DMP. The empty plasmid backbone (pPAP004) was used as negative control (-). $35 \%$ of the activity of the most active clones was chosen as threshold for clones to be classified as active (dashed line). Out of all tested clones, in total 96 (26\%) exhibited clear activity towards DMP.

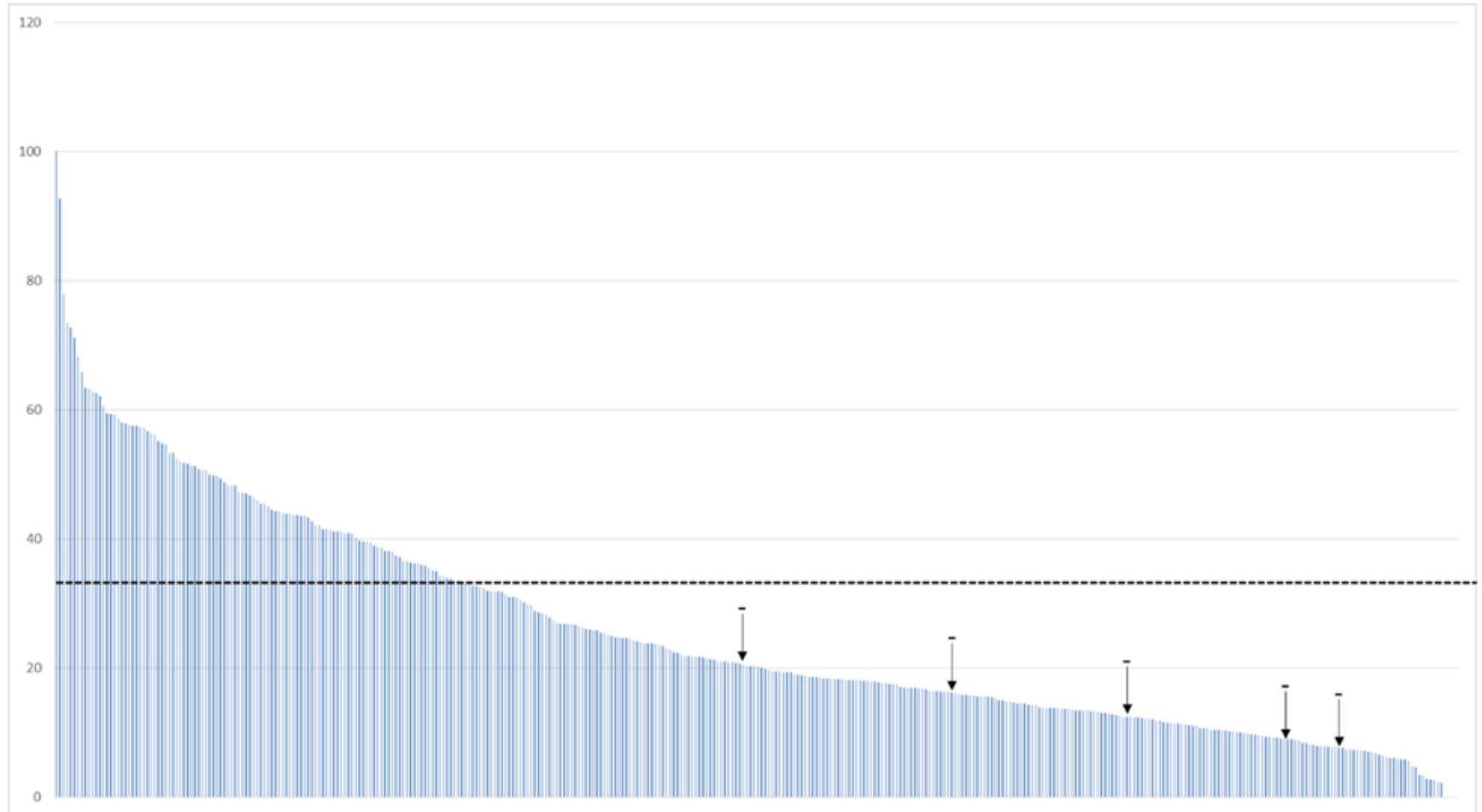




\section{Supplemental Figure 10 Screening landscape MfeUPO}

Primary screening results for the constructed promoter signal peptide shuffling library in combination with MfeUPO. 368 colonies (4 MTPs; 8 positive and negative controls respectively) were tested for NBD conversion. All clones have been ranked by their relative activity towards the substrate NBD. The empty plasmid backbone (pPAP004) was used as negative control (-). $15 \%$ of the activity of the most active clones was chosen as threshold for clones to be classified as active (dashed line). Out of all tested clones, in total 89 (24\%) exhibited clear activity towards NBD.

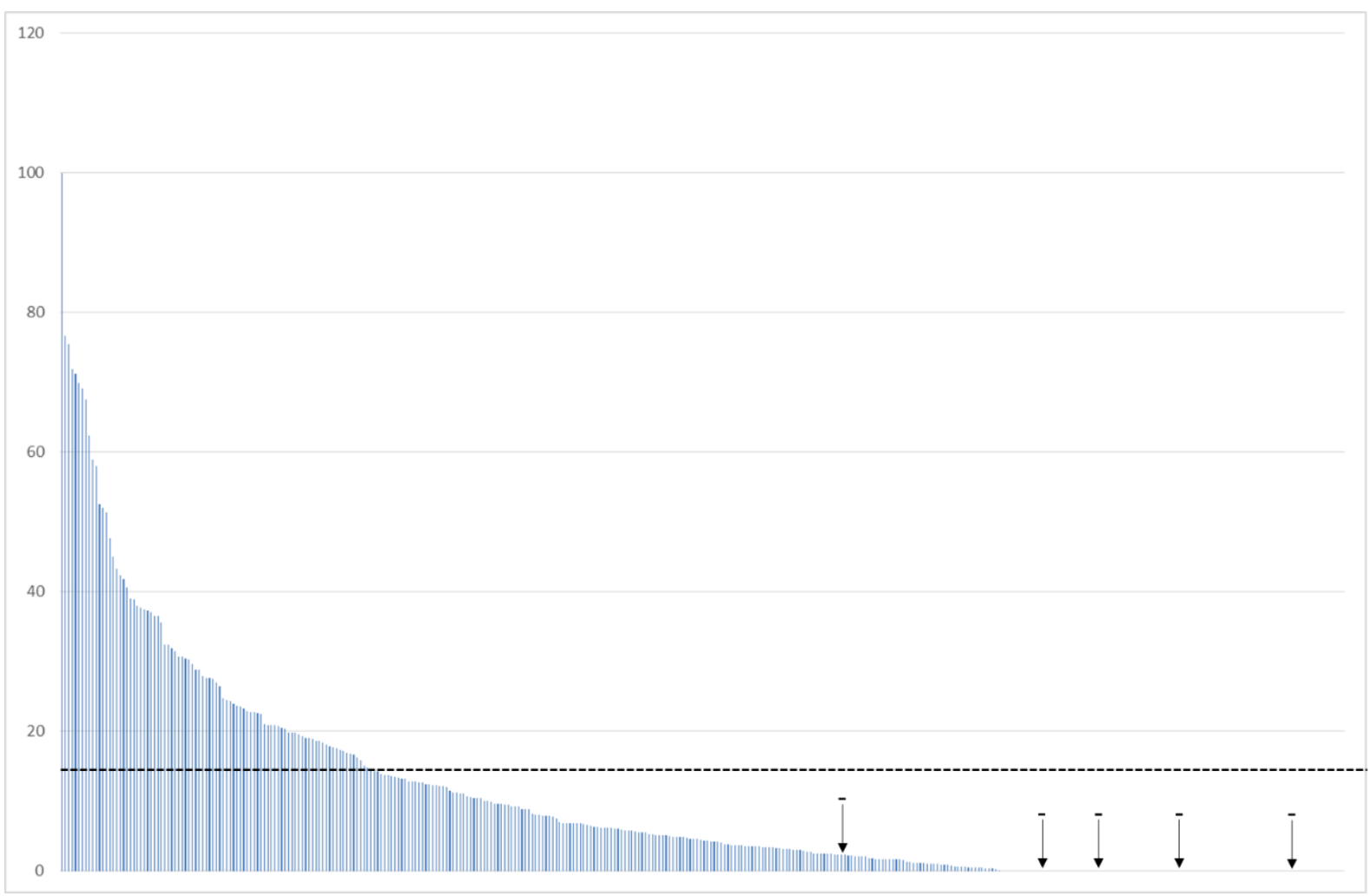




\section{Supplemental Figure 11 Screening landscape MhiUPO}

Primary screening results for the constructed promoter signal peptide shuffling library in combination with MhiUPO. 368 colonies (4 MTPs; 8 positive and negative controls respectively) were tested for NBD conversion. All clones have been ranked by their relative activity towards the substrate NBD. The empty plasmid backbone (pPAP004) was used as negative control (-) and the construct $P_{H p F M D}-G g a$-Lysozym SP-MthUPO as positive control (+). $10 \%$ of the activity of the most active clones was chosen as threshold for clones to be classified as active (dashed line). Out of all tested clones, in total 113 (31\%) exhibited clear activity towards NBD.

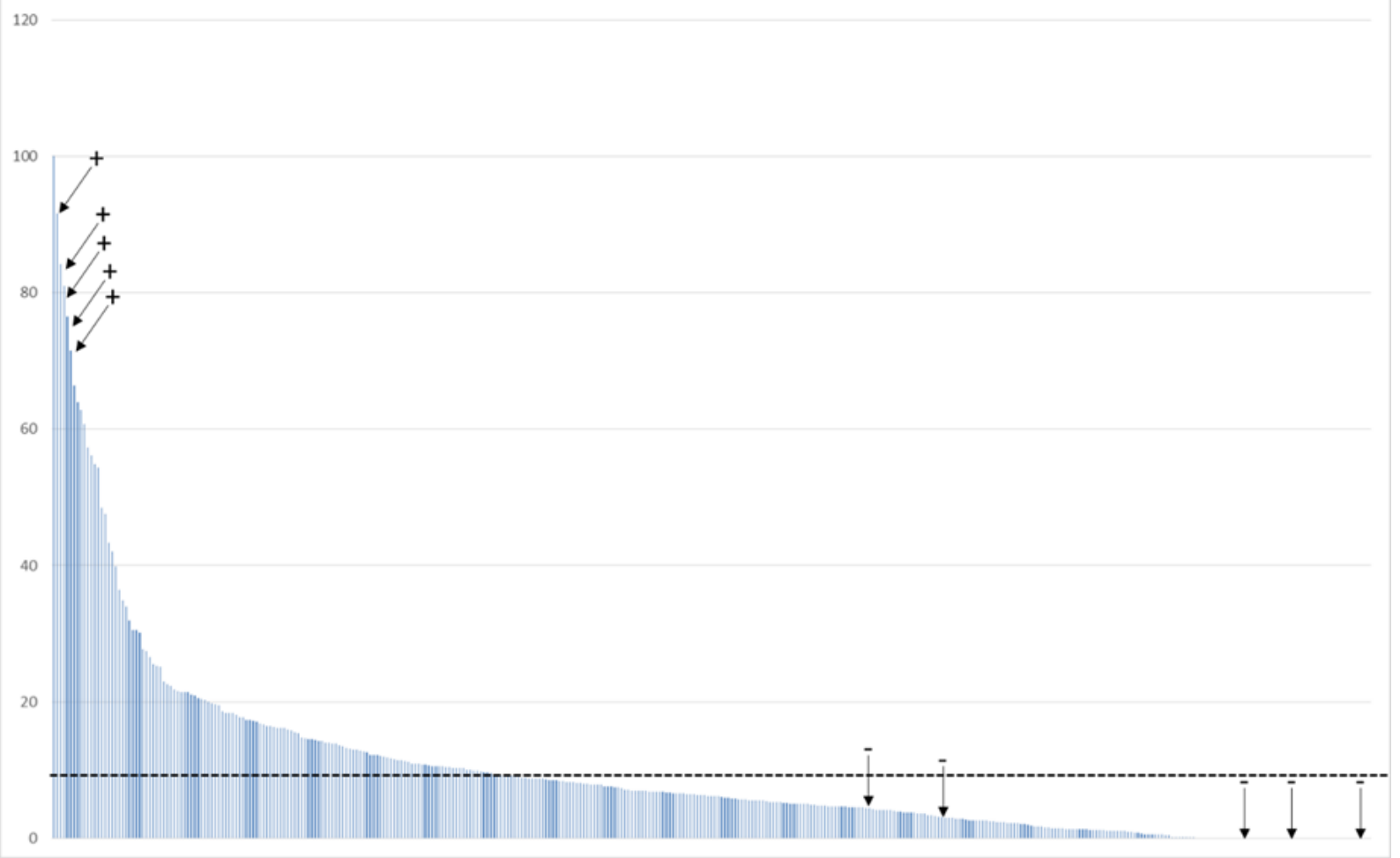


Supplemental Figure 12 Activity distribution of carbon source screening episomal/integrative constructs MhiUPO, MfeUPO, DcaUPO

6 biological replicates of the respective episomal and integrative constructs were tested towards DMP conversion. The highest sample mean within one group (out of the 12 tested conditions) was set as $100 \%$ activity and all conversion values normalized accordingly.

\section{Scheme of Boxplots:}

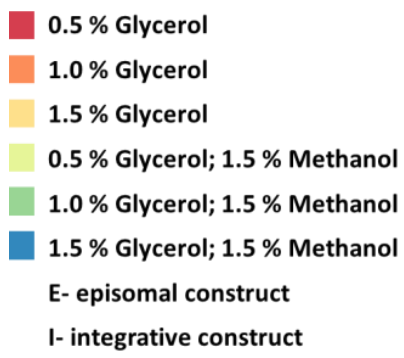

\section{MhiUPO}

$P_{P P A L D 4^{-}}$Aaw-Glucoamylase
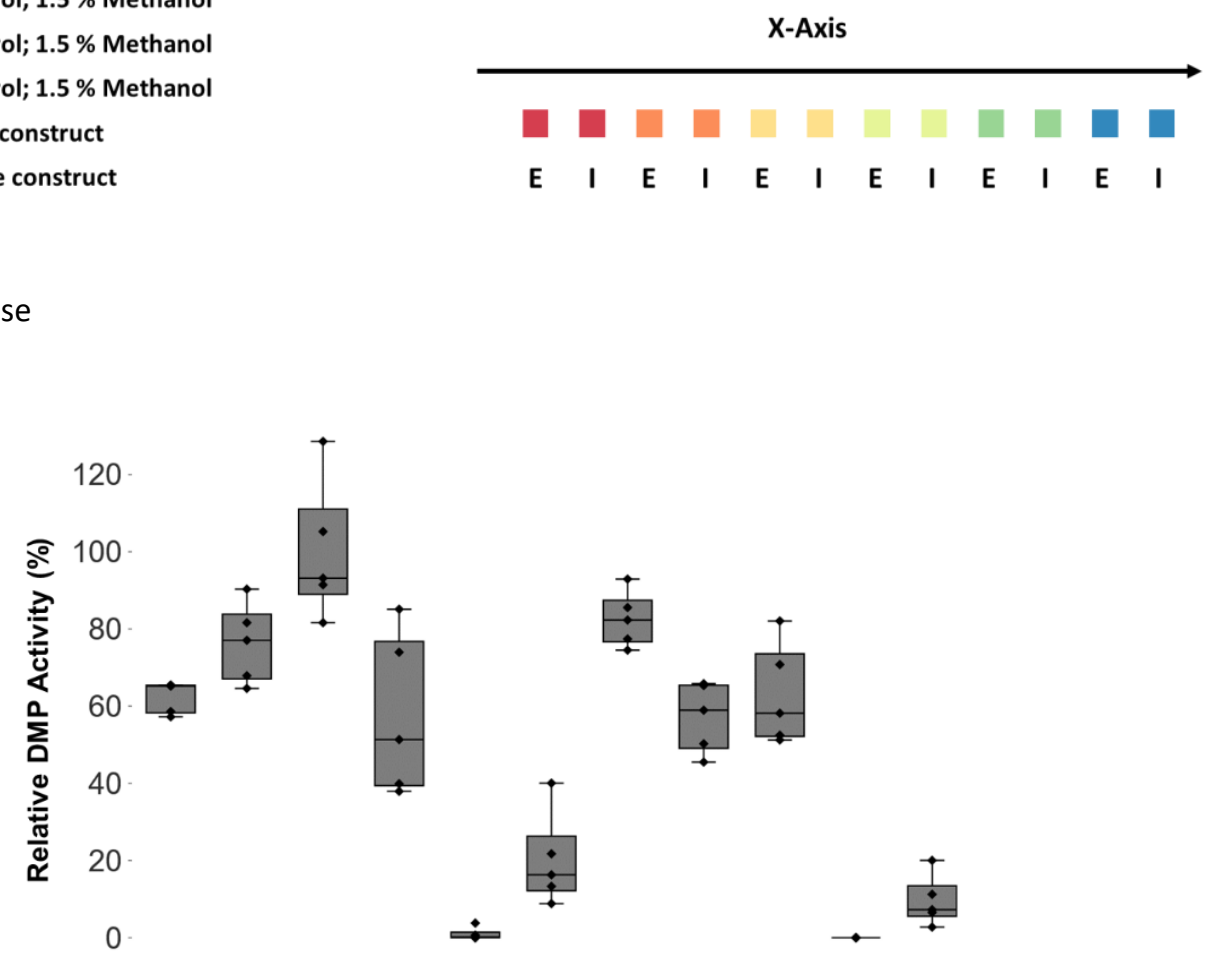

PHpMOX-Sce- $\alpha$ Galactosidase

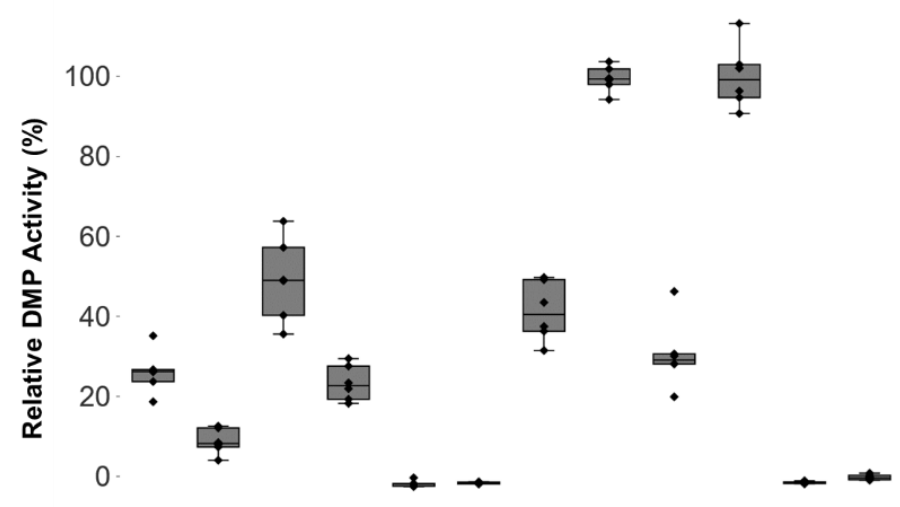




\section{MfeUPO}

$P_{\text {HpFMD- }}$ Gga-Lysozym

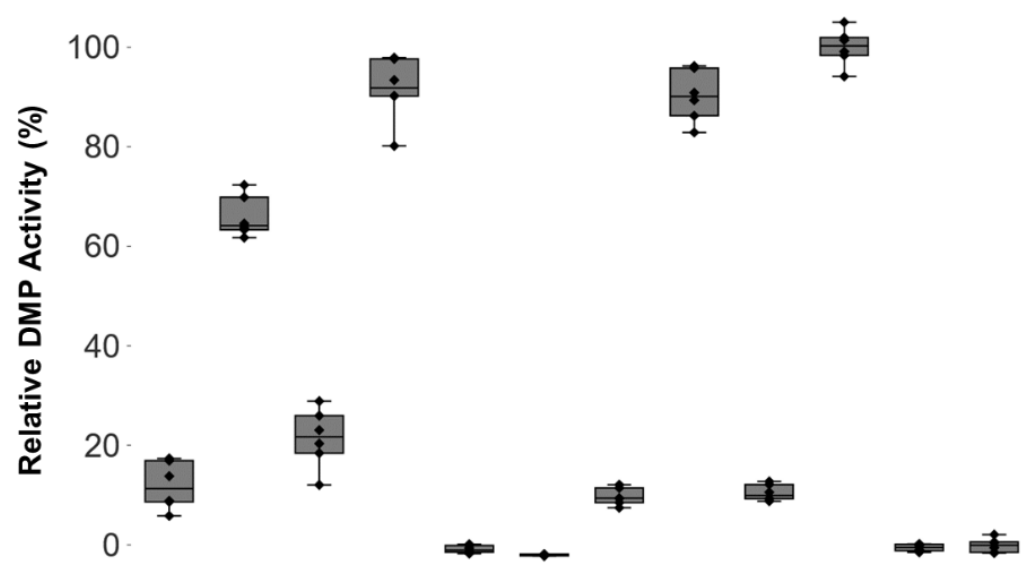

PPpFLD1- Ani-a Amylase

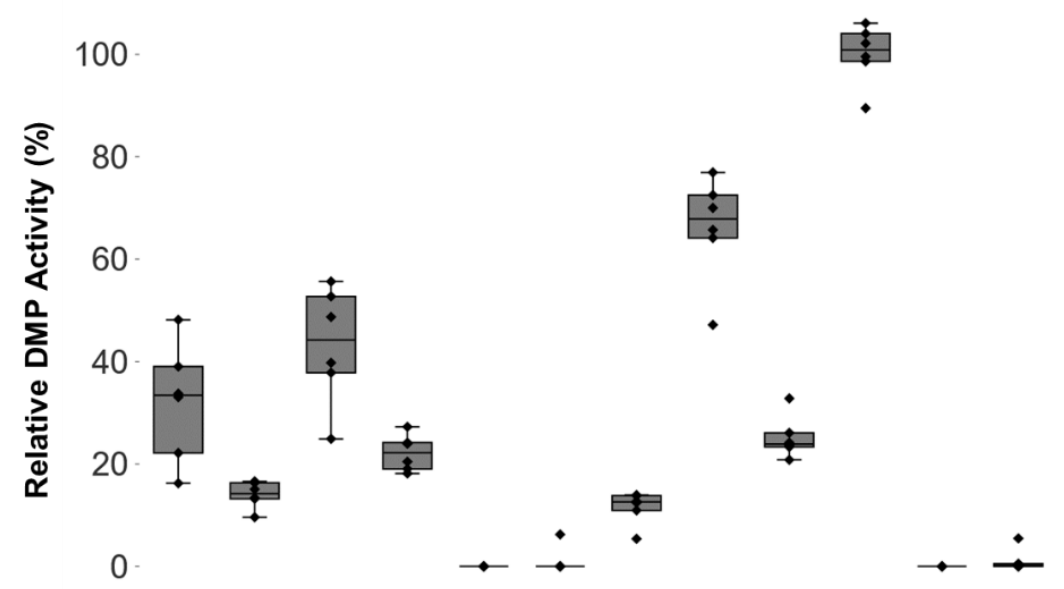




\section{DcaUPO}

Pнрмох-Sce- $\alpha$ Galactosidase

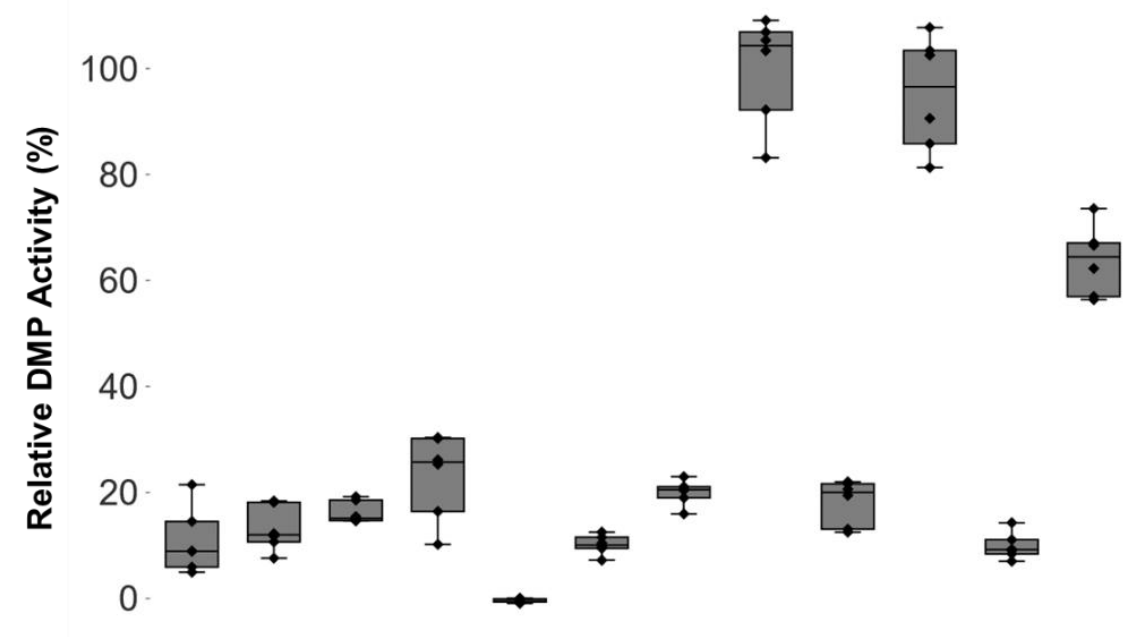

PPPFDH1- Sce- $\alpha$ Galactosidase

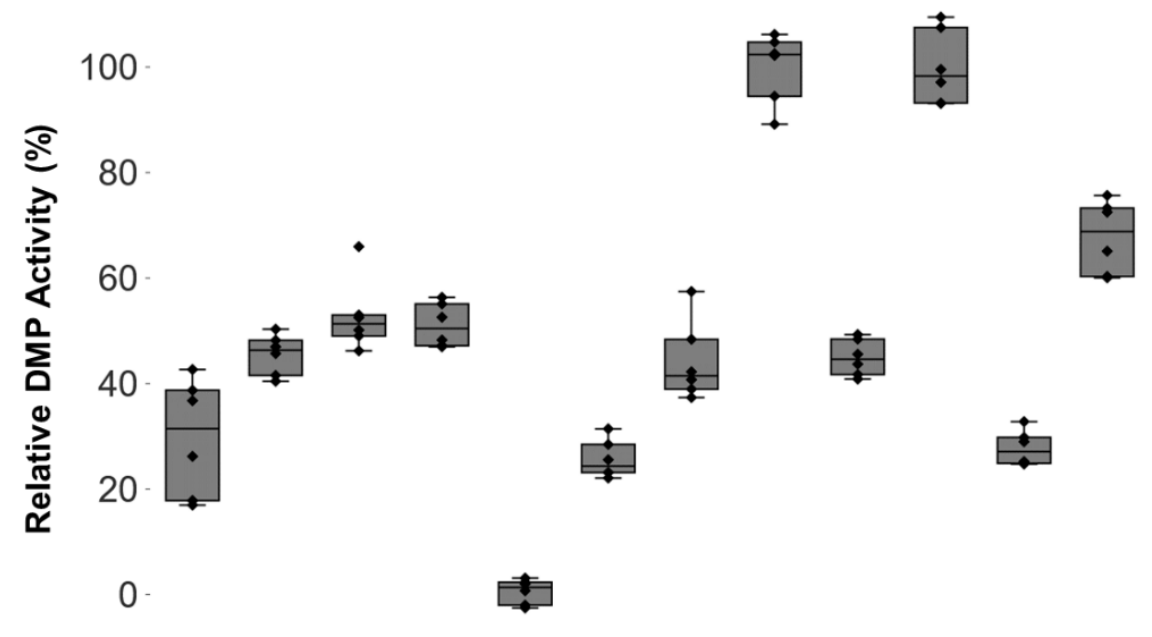




\section{Supplemental Figure 13 Optical density measurements}

8 biological replicates of the respective episomal and integrative constructs were tested regarding the final cell density. Samples of supernatant $(10 \mu \mathrm{L})$ were retrieved after $72 \mathrm{~h}$ cultivation in 96 well plates and mixed with $190 \mu \mathrm{L}$ ddH $_{2} \mathrm{O}$ prior to optical density measurements. (A): Promoter Library of TteUPO (Sce- Invertase 2 SP) compared to negative control (-) and wild type strain (X-33). (B): Carbon source variation of episomal and integrative MthUPO construct ( $P_{H p F M D}-G g a$-Lysozym) under described conditions.

(A)

\section{2}
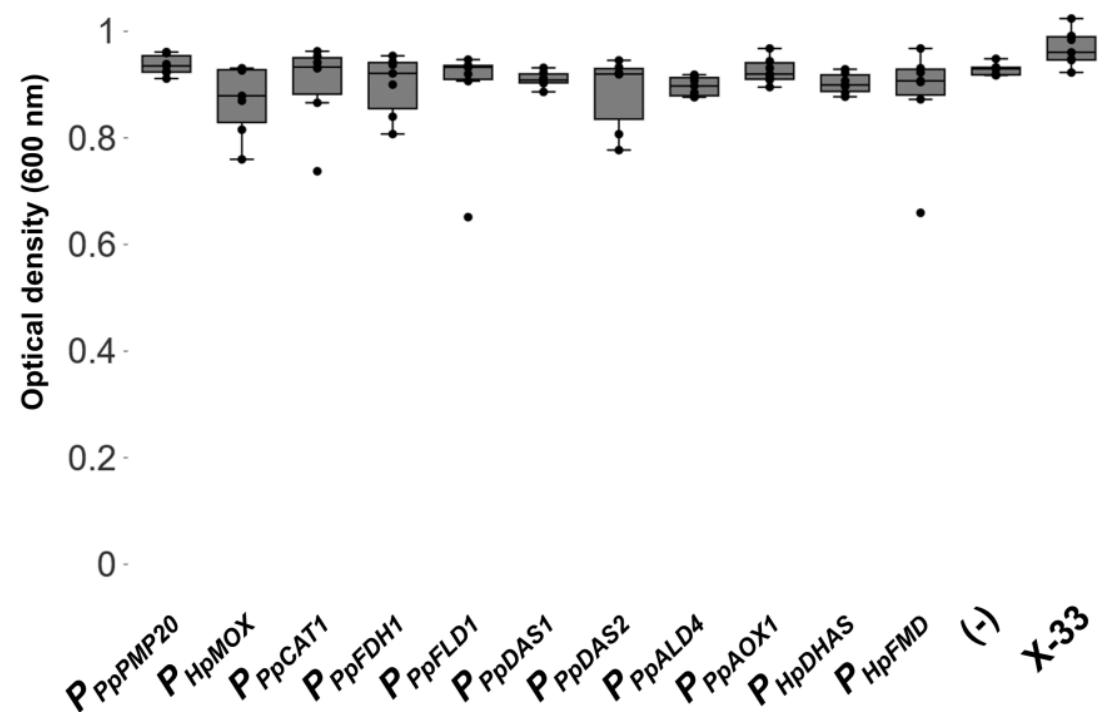

(B)

$0.5 \%$ Glycerol

$1.0 \%$ Glycerol

$1.5 \%$ Glycerol

$0.5 \%$ Glycerol; $1.5 \%$ Methanol

$1.0 \%$ Glycerol; $1.5 \%$ Methanol

$1.5 \%$ Glycerol; $1.5 \%$ Methanol

E- episomal construct

I- integrative construct
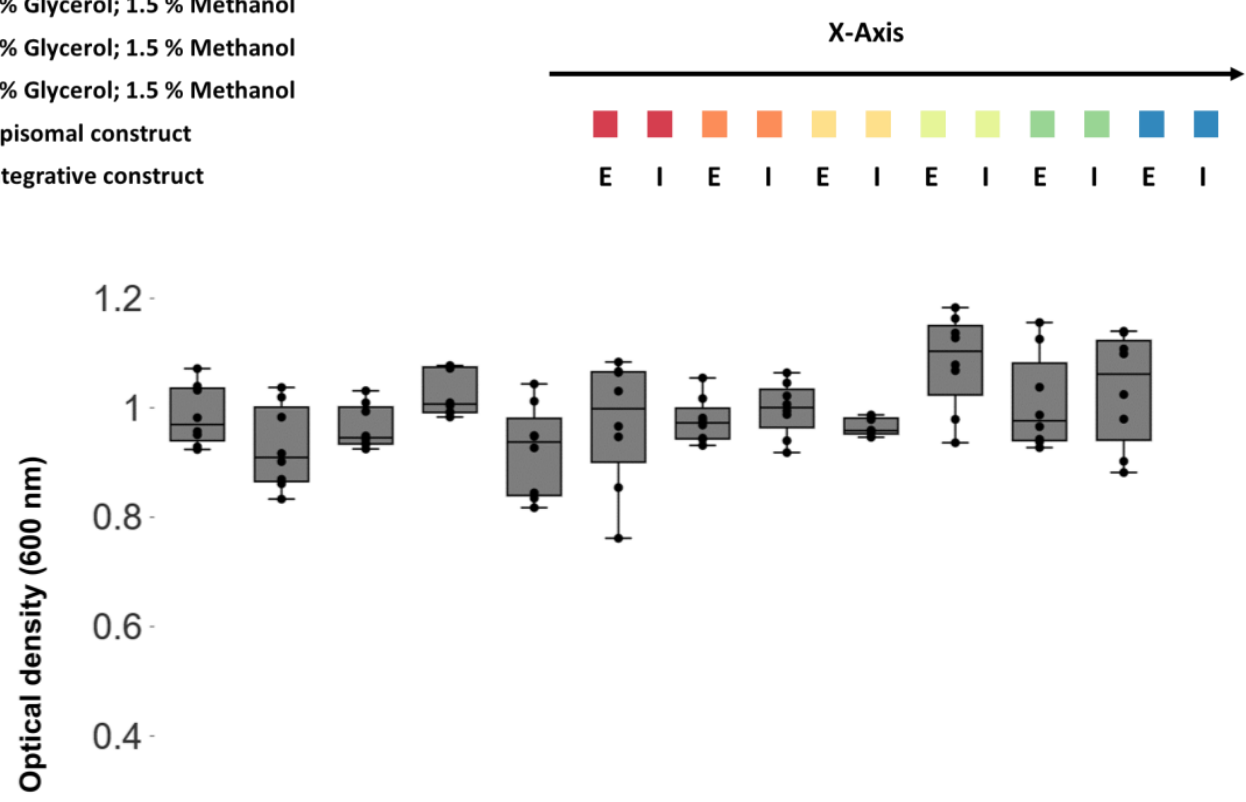

0.2 


\section{Supplemental Figure 14 Activity comparison streak-outs versus retransformation}

6 biological replicates ( $40 \mu \mathrm{L}$ supernatant sample) of the respective constructs were tested towards DMP conversion. The highest sample mean within one group was set as $100 \%$ activity and all conversion values normalized accordingly.
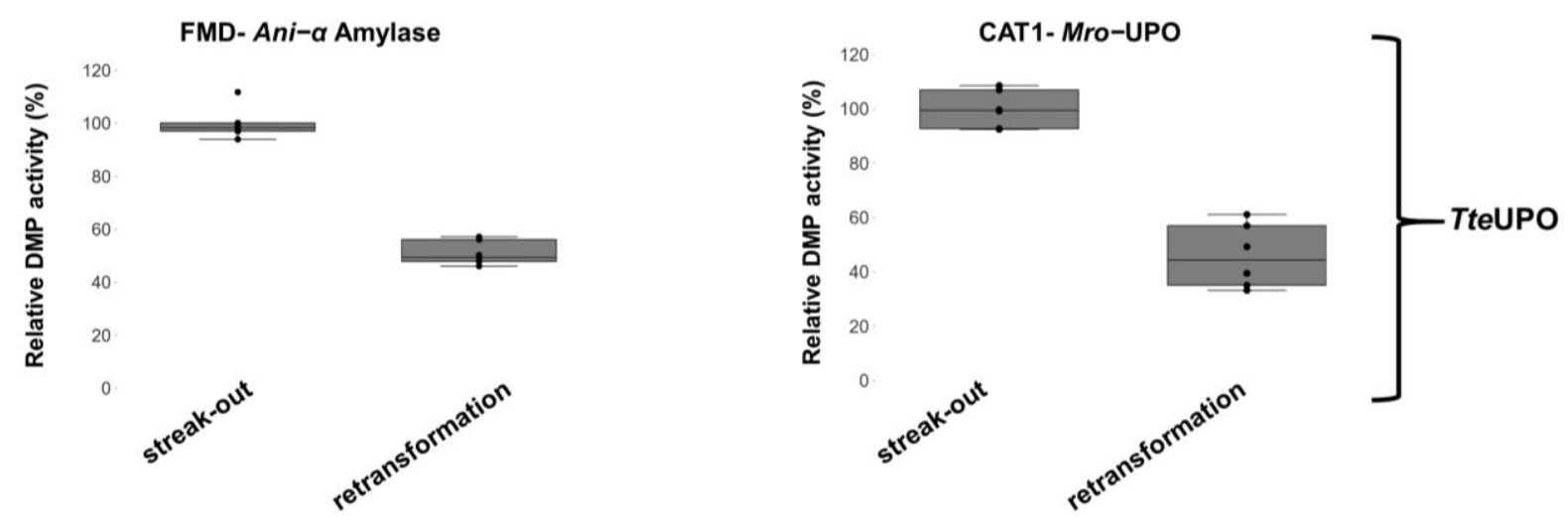

FMD- Gga-Lysozym
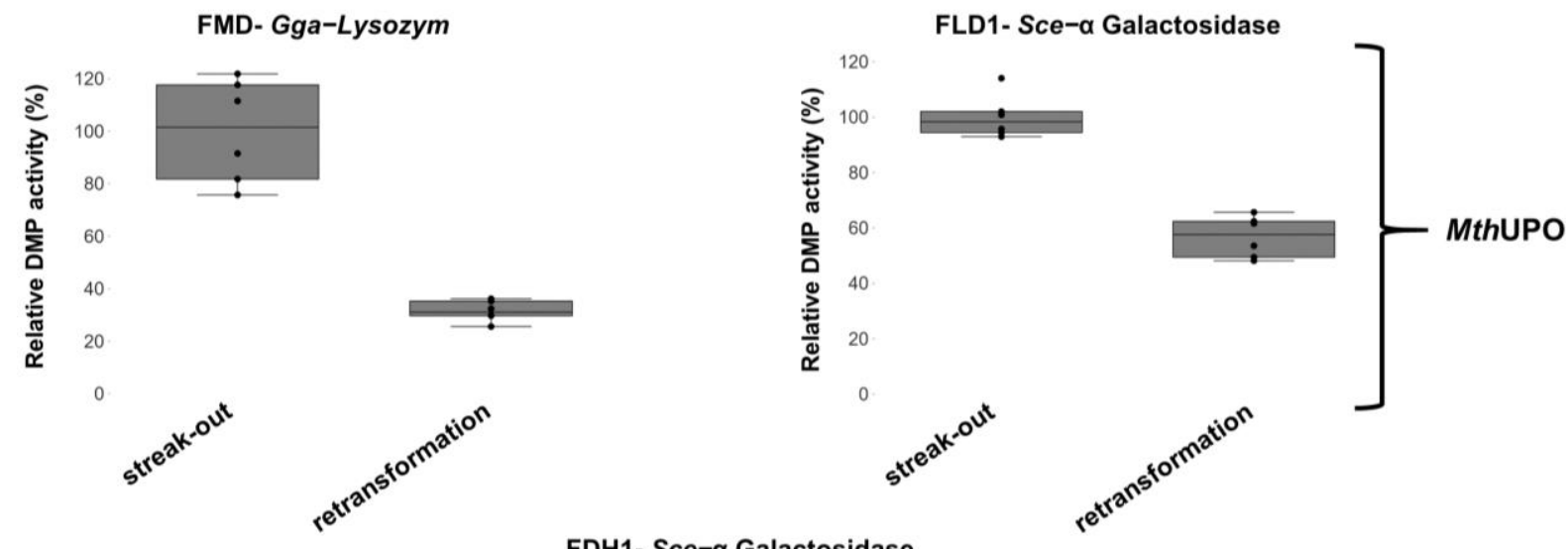

FDH1- Sce- $\alpha$ Galactosidase
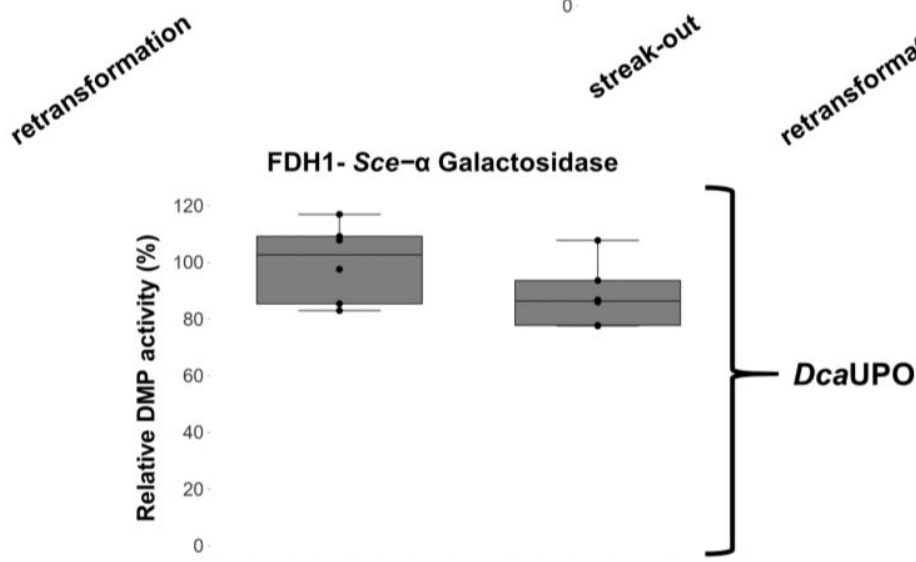


\section{Supplemental Figure 15 Evaluation of occurring standard deviations in 96 well plate screening}

96 biological replicates ( $40 \mu \mathrm{L}$ supernatant sample; 88 UPO samples (+) and negative control (-)) of the respective enzymes were tested towards DMP conversion (slope determination). The sample mean activity was set as $100 \%$ activity and all conversion values normalized accordingly. Standard deviation (SD) within the group of enzyme samples as indicated within the plots.

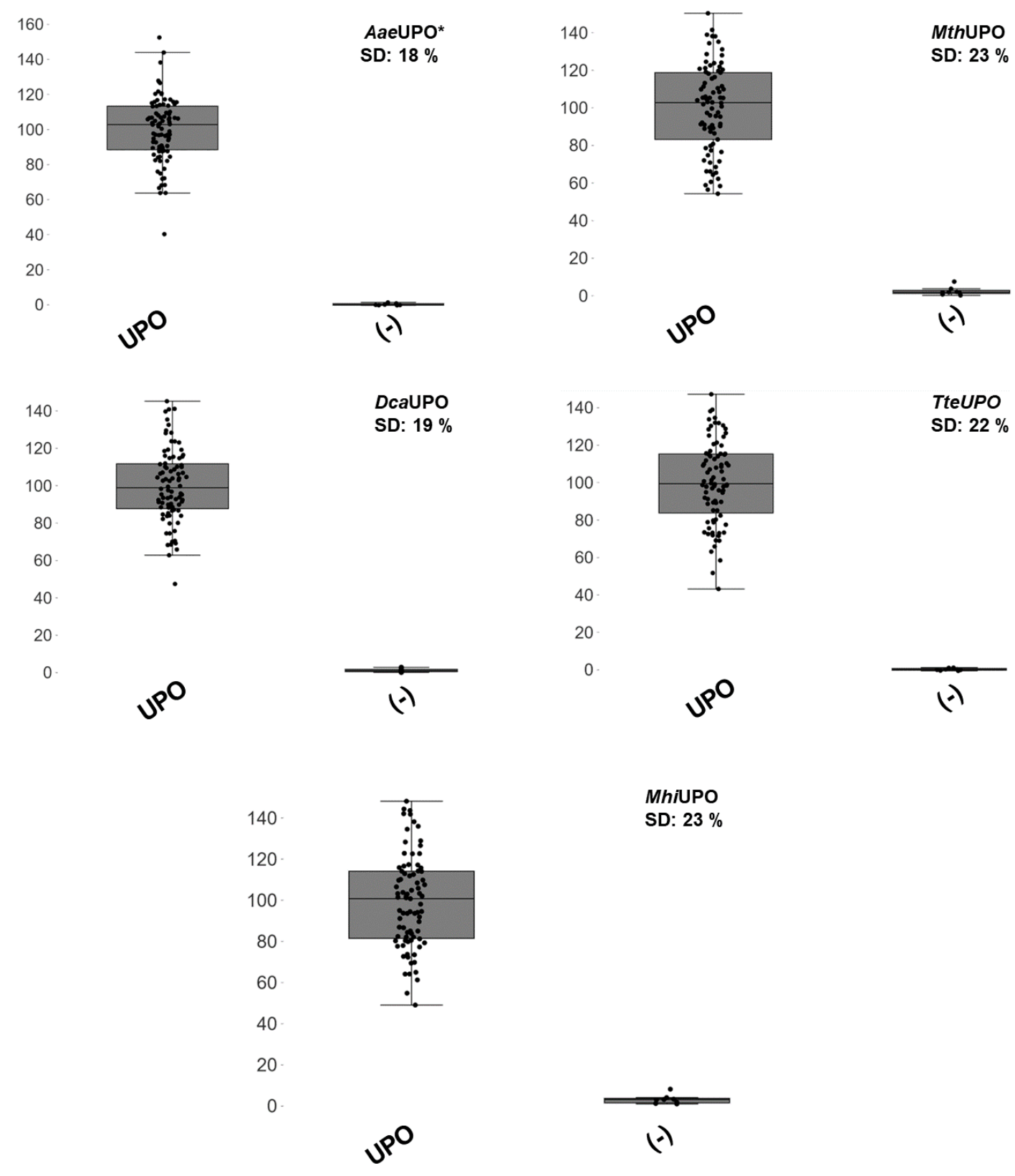




\section{Supplemental Figure 16 Activity landscapes of TteUPO error prone PCR libraries}

192 colonies (182 UPO samples and four positive (wild type TteUPO) and four negative controls (pPAP004)) per library were cultivated and respective enzymes tested towards DMP conversion $(40 \mu \mathrm{L}$ supernatant; slope determination). In each library the mean activity of the four wild type controls was set as $100 \%$ activity and all conversion values normalized accordingly. Activity landscapes ranked of the colonies ranked in a descending order and depending on the utilized $\mathrm{MnCl}_{2}$ concentration ( 0.01 to $\left.0.5 \mathrm{mM}\right)$ for library construction.

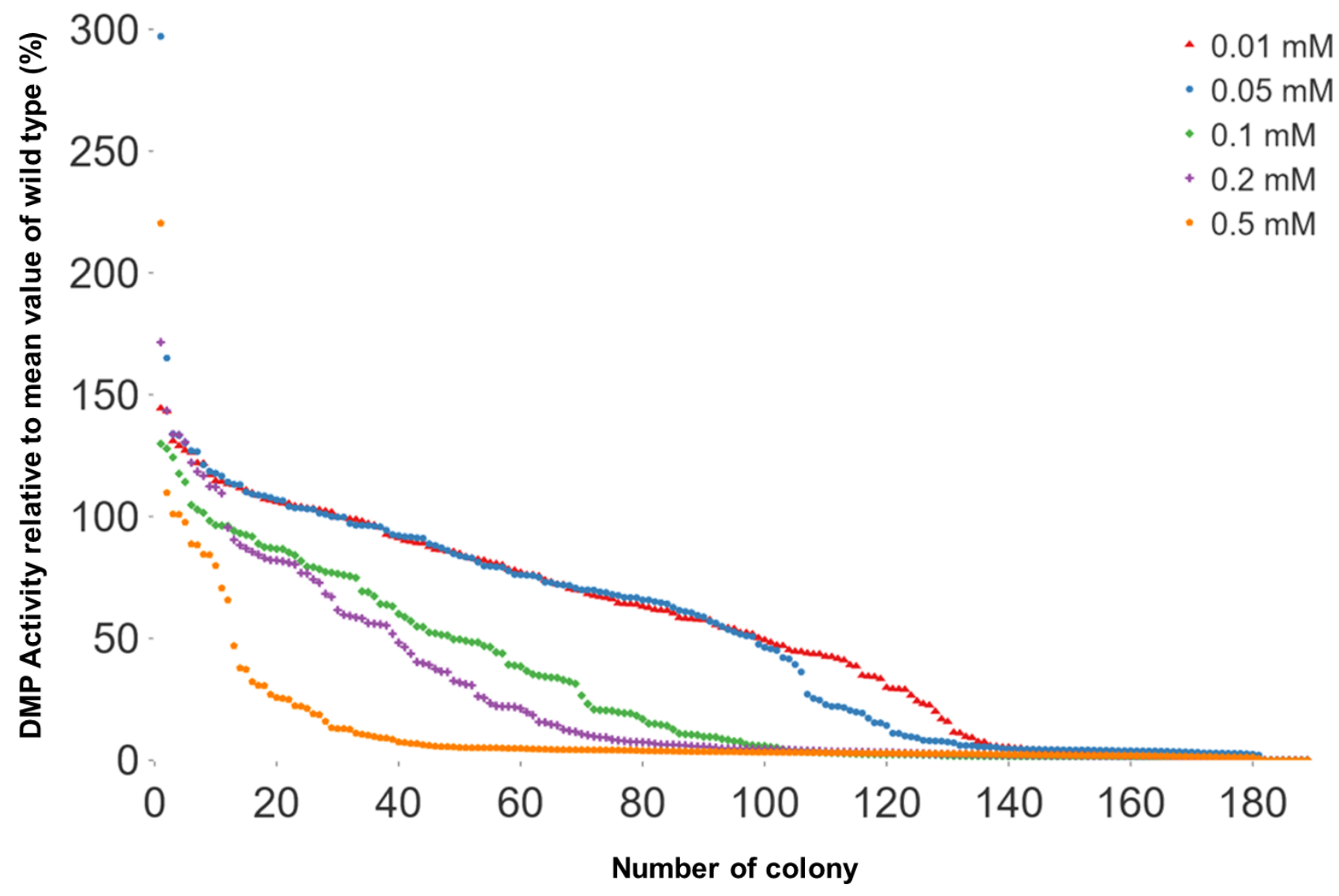




\section{Supplemental Figure 17 Absorption spectra UPOs and CO spectra}

Absorption spectra of the respective, purified UPOs were recorded in $100 \mathrm{mM}$ potassium phosphate buffer (pH 7.0) in their native state (left). The reduced, CO spectrum (right) was recorded as differential spectrum after reduction with sodium dithionite and CO treatment using the reduced sample (without $\mathrm{CO}$ treatment) as blank measurement.

\section{AaeUPO*}
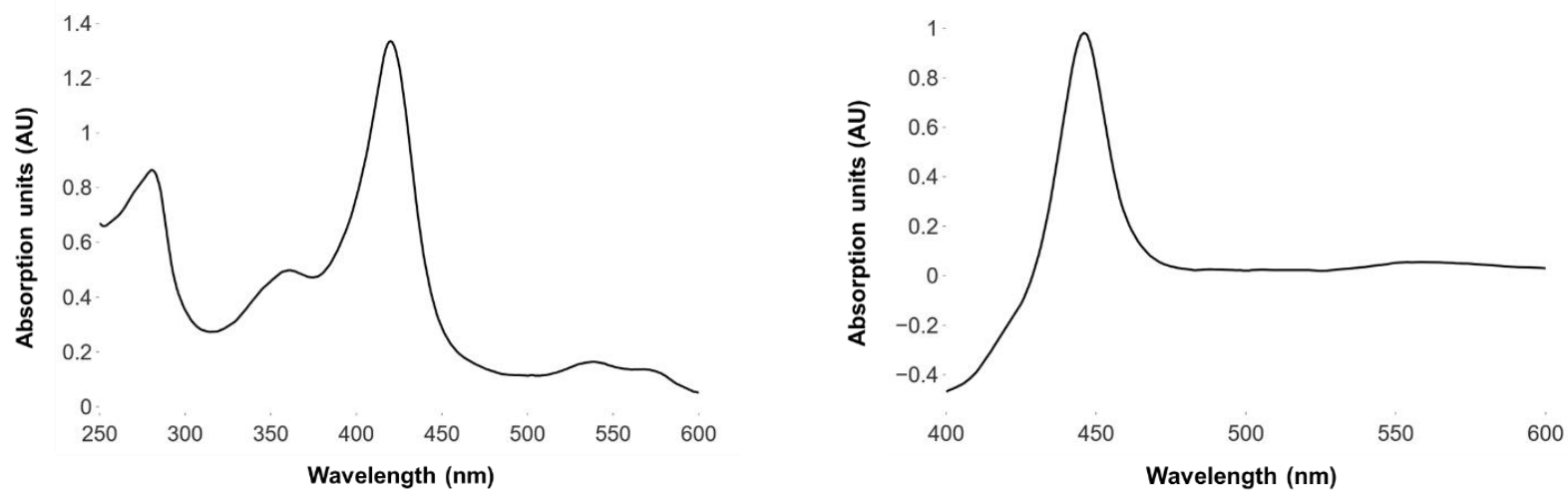

\section{TteUPO}
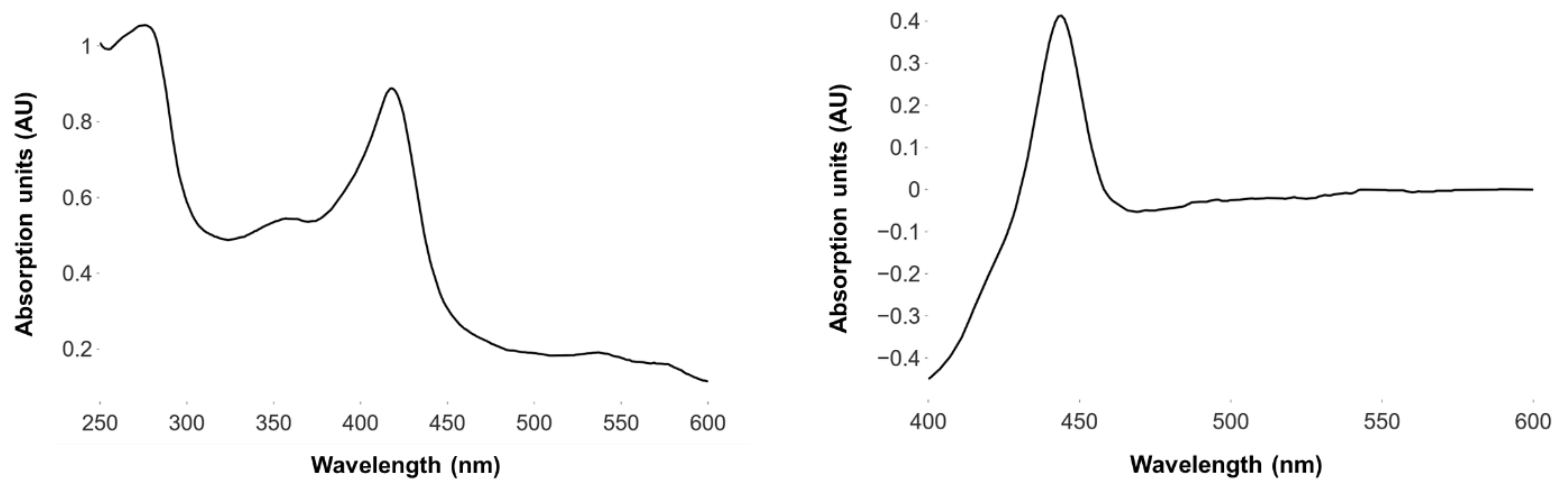

\section{MthUPO}
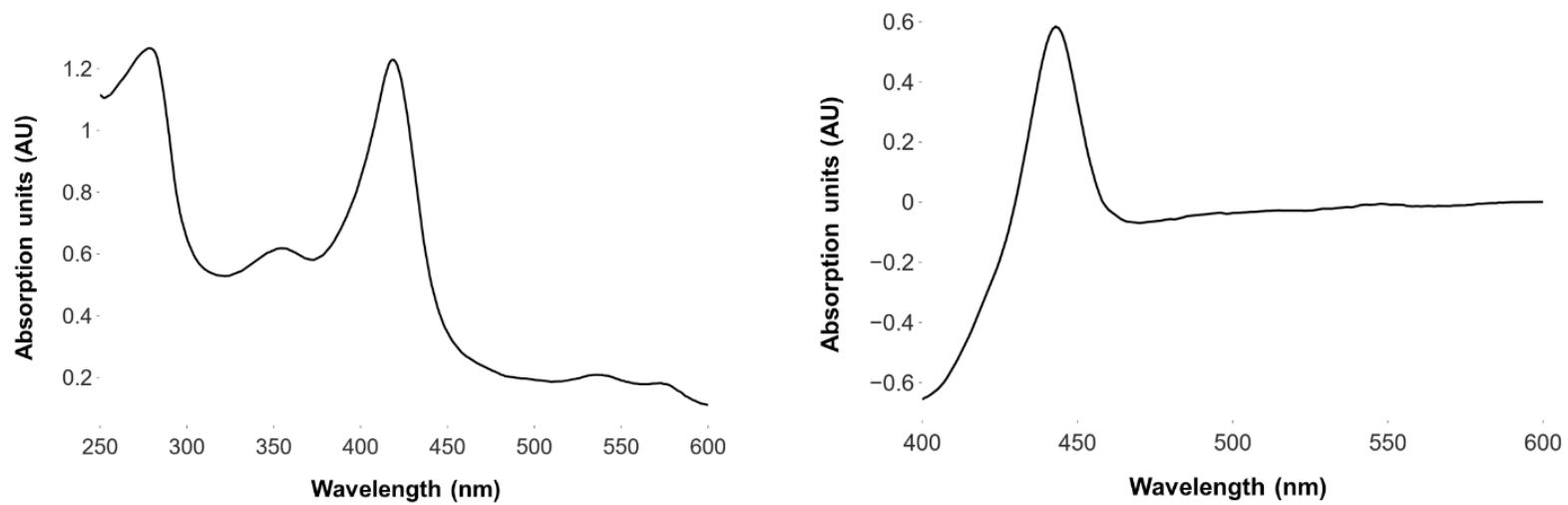


\section{DcaUPO}
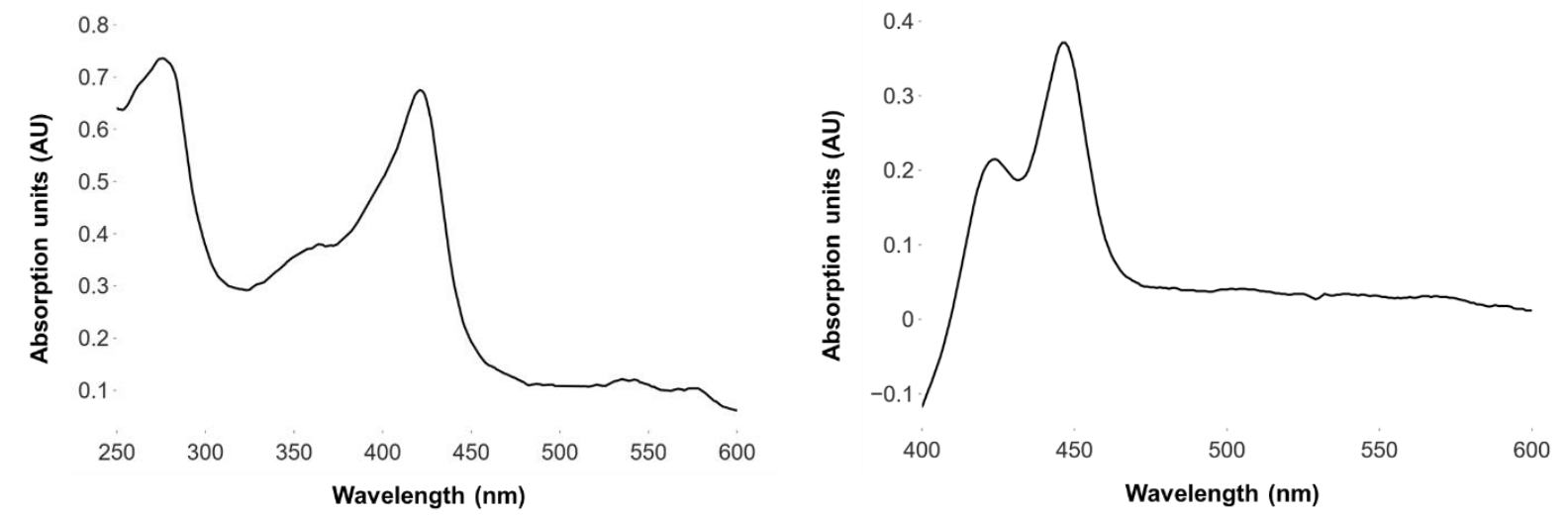

\section{Supplemental Figure 18 CO differential spectra of Myceliophthora UPOs}

Supernatant after cultivation was concentrated via ultrafiltration (approx. 10x fold concentrated) and $1 \mathrm{ml}$ samples used for measurement (in $100 \mathrm{mM}$ potassium phosphate; $\mathrm{pH}$ 6.0). Samples were reduced (+ sodium dithionite) and split into two halves- one half further treated with carbon monoxide. $\mathrm{CO}$ differential spectra were then recorded using the reduced, not $\mathrm{CO}$ treated sample as blank measurement.

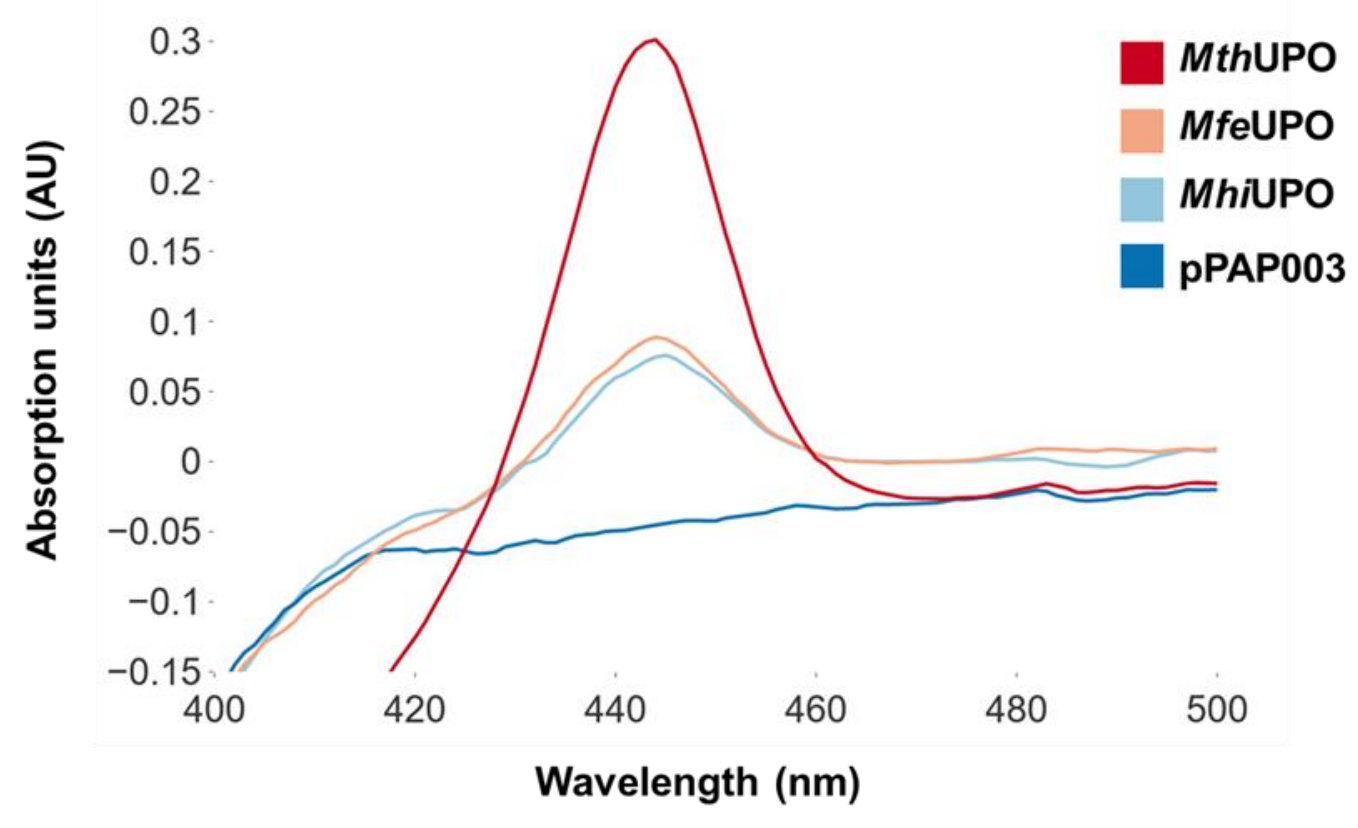




\section{Supplemental Figure 19 Screening landscape CalB}

Primary screening results for the constructed promoter signal peptide shuffling library in combination with CalB. 384 colonies (4 MTPs; 8 negative controls respectively) were tested for 4-Nitrophenyl Laurate conversion. All clones have been ranked by their relative activity towards the substrate. The empty plasmid backbone (pPAP004) was used as negative control (-). $20 \%$ of the activity of the overall achieved activity was chosen as threshold for clones to be classified as active (dashed line). Out of all tested clones, in total 165 (44 \%) exhibited clear, background distinguishable activity towards 4-Nitrophenyl laurate.

120

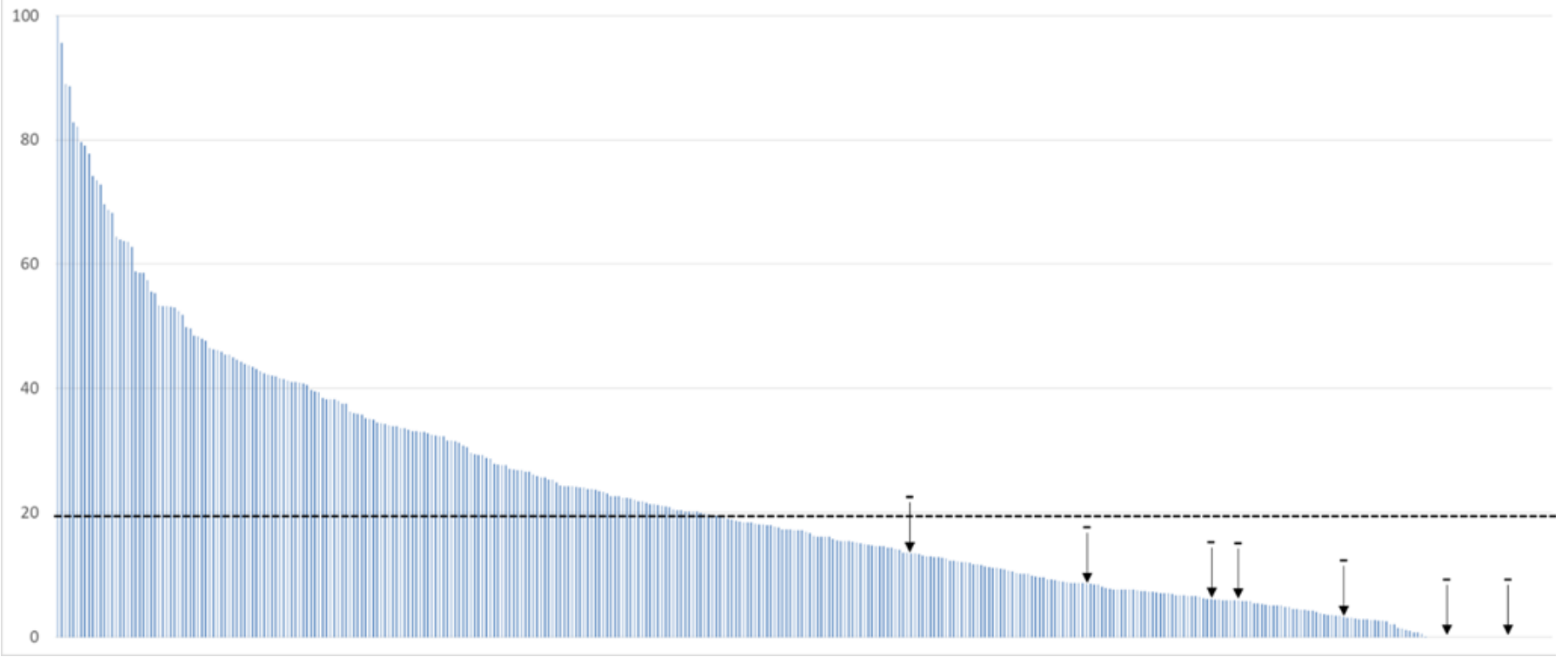




\section{Supplemental Figure 20 Screening landscape Mrl2}

Primary screening results for the constructed promoter signal peptide shuffling library in combination with Mrl2. 384 colonies (4 MTPs; 8 negative controls respectively) were tested for ABTS conversion. All clones have been ranked by their relative activity towards the substrate. The empty plasmid backbone (pPAP004) was used as negative control (-). $5 \%$ of the activity of the overall achieved activity was chosen as threshold for clones to be classified as active (dashed line). Out of all tested clones, in total 263 (70 \%) exhibited clear, background distinguishable activity towards ABTS.

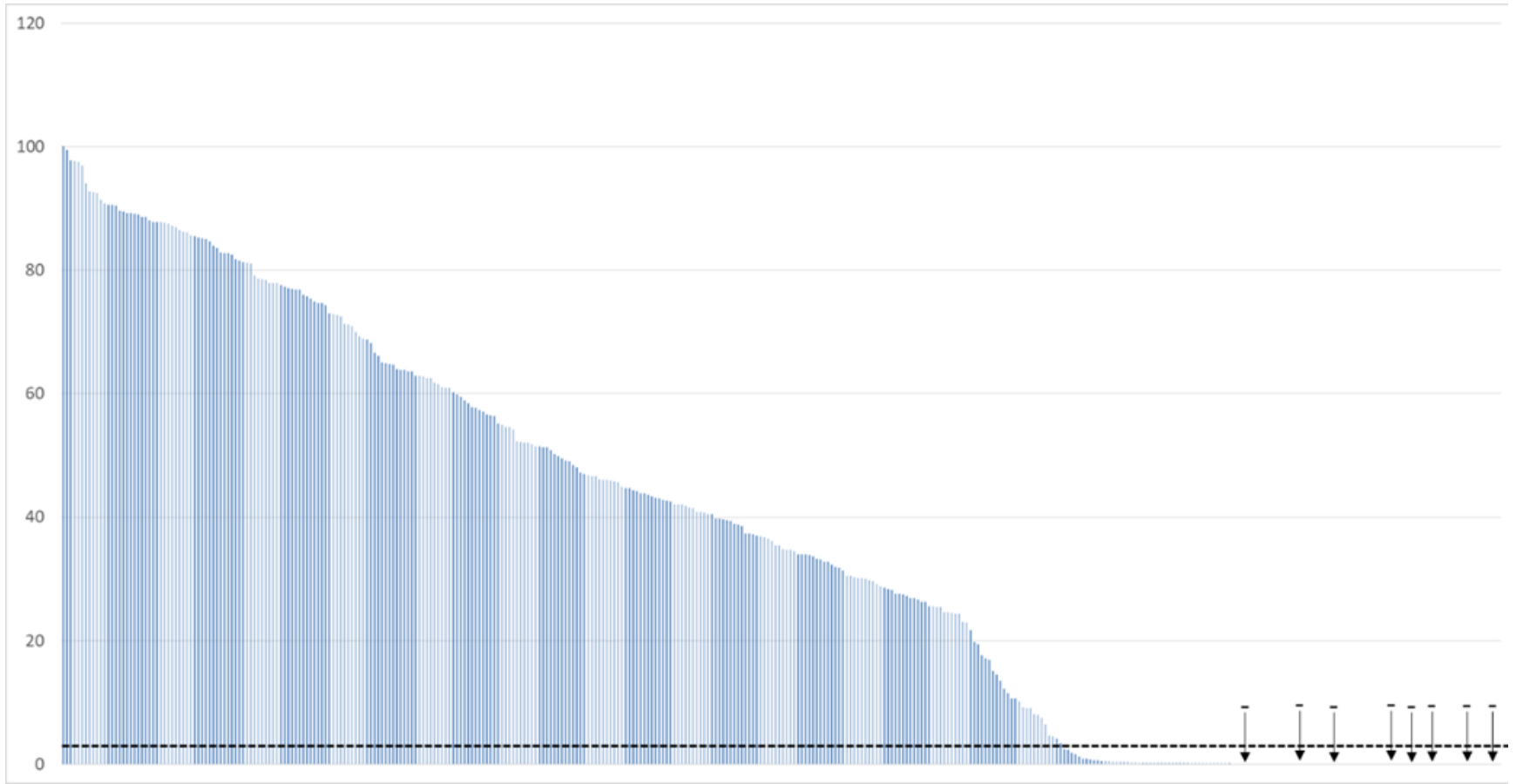


6 biological replicates of the respective episomal and integrative constructs were tested towards DMP (Mrl2) and 4-nitrophenyl laurate (CalB) conversion. The highest sample mean within one group (out of the 12 tested conditions) was set as $100 \%$ activity and all conversion values normalized accordingly.

\section{Scheme of Boxplots:}

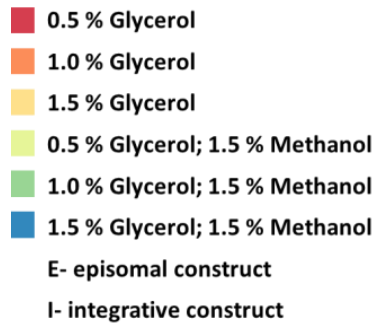

\section{CalB}

PHPFMD- Sce-Killer Protein

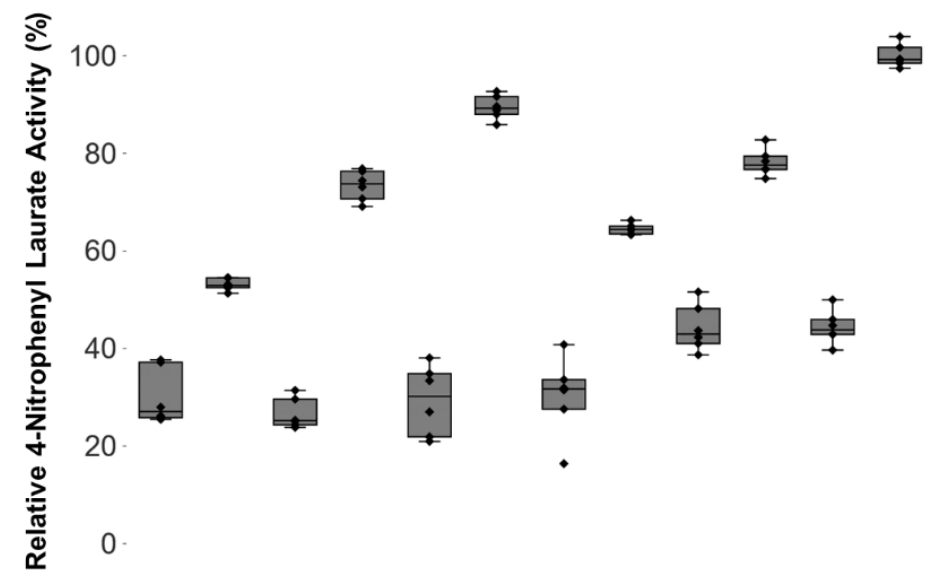

$P_{P p F L D 1}$ Ani- $\alpha$ Amylase

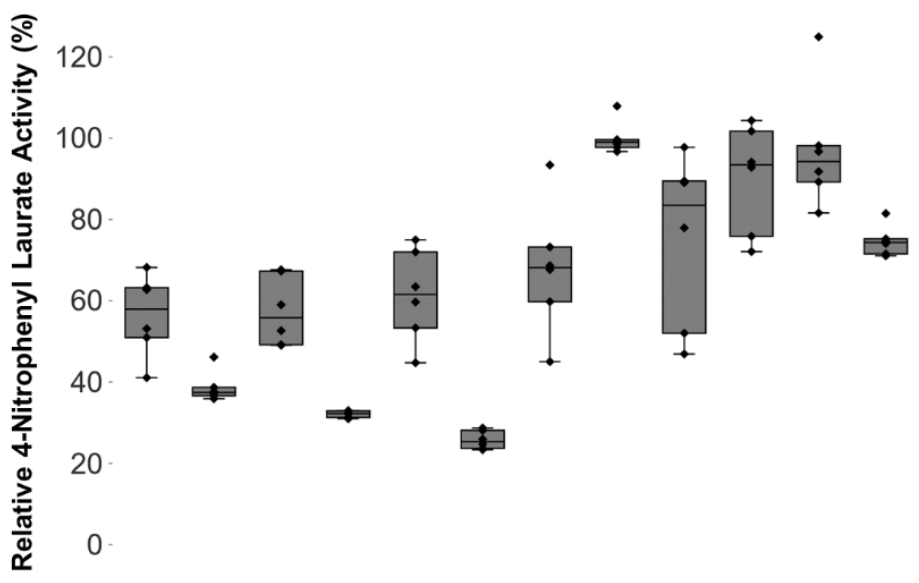


Mrl2

PHpFMD- Aaw-Glucoamylase

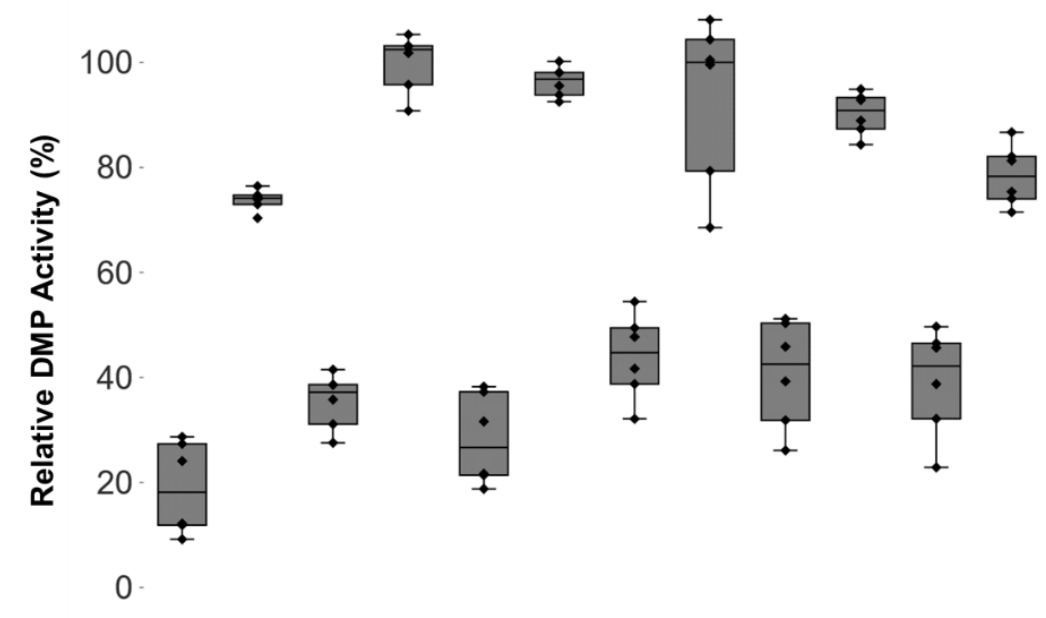

$P_{P p F L D 1}-C g l-U P O$

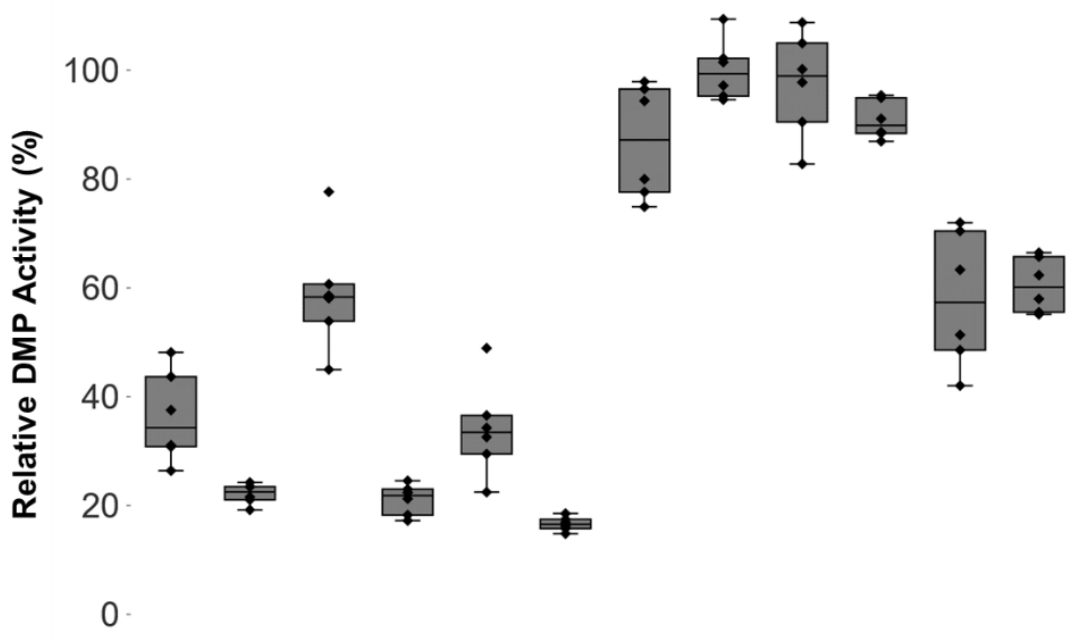


Supplemental Table 1 Overview of oligonucleotides for sequencing of the created plasmids and error prone mutagenesis

\begin{tabular}{|l|l|}
\hline Name & Sequence $\left(\mathbf{5}^{\prime} \boldsymbol{\rightarrow} \mathbf{3}^{\prime}\right.$ ) \\
\hline pAGM9121_for & CCTGTCGGGTTTCGCCACCT \\
\hline pAGM9121_rev & GCCGTTACCACCGCTGCGTT \\
\hline tGAP_rev & TCATTATGGCTGTATCTACTTTAGCGTA \\
\hline pPAP004_for & GCAACGCGGCCTTTTTACGGTTCCTGCC \\
\hline MthUPO_rev & GTATTCAACATCGGACAAGGAGCCCTAAC \\
\hline TteUPO_rev & GTGTTCAGCATTGGACAAGGACCTCTACG \\
\hline AaeUPO*_rev & CCACGAATATCGCCAGGTCGAAGCGGCTTCC \\
\hline CalB_rev & CAGGTTAAACCGGCGTCAAGCACCGAC \\
\hline Mr2Laccase_rev & GATCTTGTAAAACCATCCGGACTGACGTCTTGATTA \\
\hline MfeUPO_rev & GCCATGATTGGCCAAGGTATTTAACATTGGACA \\
\hline MhiUPO_rev & GGTTTGCCAAGGTGTTTAACATAGGACATGGAG \\
\hline DcaUPO_rev & GCCAGAGTGTTCAACATAGGGCATGGTCC \\
\hline TteUPO_erPCR_for & TTGGTCTCTAATGGTCGCTTGGTGGTCTTTGTTTCTGTAC \\
\hline TteUPO_erPCR_rev & TTGGTCTCTAAGCTCAAGTAATACCAGCAGCATTAACATATTC \\
\hline
\end{tabular}

Supplemental Table 2 Nucleotide Sequences of the MUT promoters (as cloned in pAGM9121)

Kozak Sequence

Golden Gate Assembly overhangs

P PPPMP20

AACGTTCTGGAGTGTCAAAACAGTAGTGATAAAAGGCTATGAAGGAGGTTGTCTAGGGGCTCGCGGAGGAAAGTGATTCA AACAGACCTGCCAAAAAGAGAAAAAAGAGGGAATCCCTGTTCTTTCCAATGGAAATGACGTAACTTTAACTTGAAAAATAC CCCAACCAGAAGGGTTCAAACTCAACAAGGATTGCGTAATTCCTACAAGTAGCTTAGAGCTGGGGGAGAGACAACTGAAG GCAGCTTAACGATAACGCGGGGGGATTGGTGCACGACTCGAAAGGAGGTATCTTAGTCTTGTAACCTCTTTTTTCCAGAGGC TATTCAAGATTCATAGGCGATATCGATGTGGAGAAGGGTGAACAATATAAAAGGCTGGAGAGATGTCAATGAAGCAGCTG GATAGATTTCAAATTTTCTAGATTTCAGAGTAATCGCACAAAACGAAGGAATCCCACCAAGCAAAAAAAAAAATCTAAGAT CATACAAAATG

\section{$\boldsymbol{P}_{\text {HpMOX }}$}

AACGCGACGCGGAGAACGATCTCCTCGAGCTGCTCGCGGATCAGCTTGTGGCCCGGTAATGGAACCAGGCCGACGGCACGC TCCTTGCGGACCACGGTGGCTGGCGAGCCCAGTTTGTGAACGAGGTCGTTTAGAACGTCCTGCGCAAAGTCCAGTGTCAGA TGAATGTCCTCCTCGGACCAATTCAGCATGTTCTCGAGCAGCCATCTGTCTTTGGAGTAGAAGCGTAATCTCTGCTCCTCGTT ACTGTACCGGAAGAGGTAGTTTGCCTCGCCGCCCATAATGAACAGGTTCTCTTTCTGGTGGCCTGTGAGCAGCGGGGACGTC TGGACGGCGTCGATGAGGCCCTTGAGGCGCTCGTAGTACTTGTTCGCGTCGCTGTAGCCGGCCGCGGTGACGATACCCACA TAGAGGTCCTTGGCCATTAGTTTGATGAGGTGGGGCAGGATGGGCGACTCGGCATCGAAATTTTTGCCGTCGTCGTACAGTG TGATGTCACCATCGAATGTAATGAGCTGCAGCTTGCGATCTCGGATGGTTTTGGAATGGAAGAACCGCGACATCTCCAACA GCTGGGCCGTGTTGAGAATGAGCCGGACGTCGTTGAACGAGGGGGCCACAAGCCGGCGTTTGCTGATGGCGCGGCGCTCGT CCTCGATGTAGAAGGCCTTTTCCAGAGGCAGTCTCGTGAAGAAGCTGCCAACGCTCGGAACCAGCTGCACGAGCCGAGACA ATTCGGGGGTGCCGGCTTTGGTCATTTCAATGTTGTCGTCGATGAGGAGTTCGAGGTCGTGGAAGATTTCCGCGTAGCGGCG TTTTGCCTCAGAGTTTACCATGAGGTCGTCCACTGCAGAGATGCCGTTGCTCTTCACCGCGTACAGGACGAACGGCGTGGCC AGCAGGCCCTTGATCCATTCTATGAGGCCATCTCGACGGTGTTCCTTGAGTGCGTACTCCACTCTGTAGCGACTGGACATCT CGAGACTGGGCTTGCTGTGCTGGATGCACCAATTAATTGTTGCCGCATGCATCCTTGCACCGCAAGTTTTTAAAACCCACTC GCTTTAGCCGTCGCGTAAAACTTGTGAATCTGGCAACTGAGGGGGTTCTGCAGCCGCAACCGAACTTTTCGCTTCGAGGACG CAGCTGGATGGTGTCATGTGAGGCTCTGTTTGCTGGCGTAGCCTACAACGTGACCTTGCCTAACCGGACGGCGCTACCCACT GCTGTCTGTGCCTGCTACCAGAAAATCACCAGAGCAGCAGAGGGCCGATGTGGCAACTGGTGGGGTGTCGGACAGGCTGTT TCTCCACAGTGCAAATGCGGGTGAACCGGCCAGAAAGTAAATTCTTATGCTACCGTGCAGTGACTCCGACATCCCCAGTTTT TGCCCTACTTGATCACAGATGGGGTCAGCGCTGCCGCTAAGTGTACCCAACCGTCCCCACACGGTCCATCTATAAATACTGC TGCCAGTGCACGGTGGTGACATCAATCTAAAGTACAAAAACAAATCATACAAAATG 


\section{PPPFLDI}

AACGGCATGCAGGAATCTCTGGCACGGTGCTAATGGTAGTTATCCAACGGAGCTGAGGTAGTCGATATATCTGGATATGCC GCCTATAGGATAAAAACAGGAGAGGGTGAACCTTGCTTATGGCTACTAGATTGTTCTTGTACTCTGAATTCTCATTATGGGA AACTAAACTAATCTCATCTGTGTGTTGCAGTACTATTGAATCGTTGTAGTATCTACCTGGAGGGCATTCCATGAATTAGTGA GATAACAGAGTTGGGTAACTAGAGAGAATAATAGACGTATGCATGATTACTACACAACGGATGTCGCACTCTTTCCTTAGT TAAAACTATCATCCAATCACAAGATGCGGGCTGGAAAGACTTGCTCCCGAAGGATAATCTTCTGCTTCTATCTCCCTTCCTC ATATGGTTTCGCAGGGCTCATGCCCCTTCTTCCTTCGAACTGCCCGATGAGGAAGTCCTTAGCCTATCAAAGAATTCGGGAC CATCATCGATTTTTAGAGCCTTACCTGATCGCAATCAGGATTTCACTACTCATATAAATACATCGCTCAAAGCTCCAACTTTG CTTGTTCATACAATTCTTGATATTCACATCATACAAAATG

\section{PPPFDI}

AACGAAATGGCAGAAGGATCAGCCTGGACGAAGCAACCAGTTCCAACTGCTAAGTAAAGAAGATGCTAGACGAAGGAGAC TTCAGAGGTGAAAAGTTTGCAAGAAGAGAGCTGCGGGAAATAAATTTTCAATTTAAGGACTTGAGTGCGTCCATATTCGTG TACGTGTCCAACTGTTTTCCATTACCTAAGAAAAACATAAAGATTAAAAAGATAAACCCAATCGGGAAACTTTAGCGTGCC GTTTCGGATTCCGAAAAACTTTTGGAGCGCCAGATGACTATGGAAAGAGGAGTGTACCAAAATGGCAAGTCGGGGGCTACT CACCGGATAGCCAATACATTCTCTAGGAACCAGGGATGAATCCAGGTTTTTGTTGTCACGGTAGGTCAAGCATTCACTTCTT AGGAATATCTCGTTGAAAGCTACTTGAAATCCCATTGGGTGCGGAACCAGCTTCTAATTAAATAGTTCGATGATGTTCTCTA AGTGGGACTCTACGGCTCAAACTTCTACACAGCATCATCTTAGTAGTCCCTTCCCAAAACACCATTCTAGGTTTCGGAACGT AACGAAACAATGTTCCTCTCTTCACATTGGGCCGTTACTCTAGCCTTCCGAAGAACCAATAAAAGGGACCGGCTGAAACGG GTGTGGAAACTCCTGTCCAGTTTATGGCAAAGGCTACAGAAATCCCAATCTTGTCGGGATGTTGCTCCTCCCAAACGCCATA TTGTACTGCAGTTGGTGCGCATTTTAGGGAAAATTTACCCCAGATGTCCTGATTTTCGAGGGCTACCCCCAACTCCCTGTGCT TATACTTAGTCTAATTCTATTCAGTGTGCTGACCTACACGTAATGATGTCGTAACCCAGTTAAATGGCCGAAAAACTATTTA AGTAAGTTTATTTCTCCTCCAGATGAGACTCTCCTTCTTTTCTCCGCTAGTTATCAAACTATAAACCTATTTTACCTCAAATA CCTCCAACATCACCCACTTAAACATCATACAAAATG

\section{PPPDASI}

AACGAATGATATAAACAACAATTGAGTGACAGGTCTACTTTGTTCTCAAAAGGCCATAACCATCTGTTTGCATCTCTTATCA CCACACCATCCTCCTCATCTGGCCTTCAATTGTGGGGAACAACTAGCATCCCAACACCAGACTAACTCCACCCAGATGAAAC CAGTTGTCGCTTACCAGTCAATGAATGTTGAGCTAACGTTCCTTGAAACTCGAATGATCCCAGCCTTGCTGCGTATCATCCC TCCGCTATTCCGCCGCTTGCTCCAACCATGTTTCCGCCTTTTTCGAACAAGTTCAAATACCTATCTTTGGCAGGACTTTTCCT CCTGCCTTTTTTAGCCTCAGTTCTCGGTTAGCCTCTAGGCAAATTCTGTTCTTCATACCTATATCAACTTTTCATCAGATAGCC TTTGGGTTCAAAAAAGAACTAAAGCAGGATGCCTGATATATAAATCCCAGATGATCTGCTTTTGAAACTATTTTCAGTATCT TGATTCGTTTACTTACAAACAACTATTGTTGATTTTATCTGGAGAATAATCGAACATCATACAAAATG

\section{$P_{P P D A S 2}$}

AACGCAATCTGTGGTTTGCTAAACTGGAAGTCTGGTAAGGACTCTAGCAAGTCCGTTACTCAAAAAGTCATACCAAGTAAG ATTACGTAACACCTGGGCATGACTTTCTAAGTTAGCAAGTCACCAAGAGGGTCCTATTTAACGTTTGGCGGTATCTGAAACA CAAGACTTGCCTATCCCATAGTACATCATATTACCTGTCAAGCTATGCTACCCCACAGAAATACCCCAAAAGTTGAAGTGAA AAAATGAAAATTACTGGTAACTTCACCCCATAACAAACTTAATAATTTCTGTAGCCAATGAAAGTAAACCCCATTCAATGTT CCGAGATTTAGTATACTTGCCCCTATAAGAAACGAAGGATTTCAGCTTCCTTACCCCATGAACAGAAATCTTCCATTTACCC CCCACTGGAGAGATCCGCCCAAACGAACAGATAATAGAAAAAAGAAATTCGGACAAATAGAACACTTTCTCAGCCAATTA AAGTCATTCCATGCACTCCCTTTAGCTGCCGTTCCATCCCTTTGTTGAGCAACACCATCGTTAGCCAGTACGAAAGAGGAAA CTTAACCGATACCTTGGAGAAATCTAAGGCGCGAATGAGTTTAGCCTAGATATCCTTAGTGAAGGGTTGTTCCGATACTTCT CCACATTCAGTCATAGATGGGCAGCTTTGTTATCATGAAGAGACGGAAACGGGCATTAAGGGTTAACCGCCAAATTATATA AAGACAACATGTCCCCAGTTTAAAGTTTTTCTTTCCTATTCTTGTATCCTGAGTGACCGTTGTGTTTAATATAACAAGTTCGT TTTAACTTAAGACCAAAACCAGTTACAACAAATTATAACCCCTCTAAACACTAAAGTTCACTCTTATCAAACTATCAAACAT CAAAATCATACAAAATG

\section{PPpCATI}

AACGTAATCGAACTCCGAATGCGGTTCTCCTGTAACCTTAATTGTAGCATAGATCACTTAAATAAACTCATGGCCTGACATC TGTACACGTTCTTATTGGTCTTTTAGCAATCTTGAAGTCTTTCTATTGTTCCGGTCGGCATTACCTAATAAATTCGAATCGAG ATTGCTAGTACCTGATATCATATGAAGTAATCATCACATGCAAGTTCCATGATACCCTCTACTAATGGAATTGAACAAAGTT TAAGCTTCTCGCACGAGTCCGAATCCATACTATGCACCCCTCAAAGTTGGGATTAGTCAGGAAAGCTGAGCAATTAACTTCC CTCGATTGGCCTGGACTTTTCGCTTAGCCTGCCGCAATCGGTAAGTTTCATTATCCCAGCGGGGTGATAGCCTCTGTTGCTCA TCAGGCCAAAATCATATATAAGCTGTAGACCCAGCACTTCAATTACTTGAAATTCACCATAACACTTGCTCTAGTCAAGACT TACAATTAAATCATACAAAATG 
AACGAGACGAAAGGTTGAATGAAACCTTTTTGCCATCCGACATCCACAGGTCCATTCTCACACATAAGTGCCAAACGCAAC AGGAGGGGATACACTAGCAGCAGACCGTTGCAAACGCAGGACCTCCACTCCTCTTCTCCTCAACACCCACTTTTGCCATCGA AAAACCAGCCCAGTTATTGGGCTTGATTGGAGCTCGCTCATTCCAATTCCTTCTACTAGGCTACTAACACCGTGACTTTATTA GCCTGTCTATCCTGGCCCCCCTGGCGAGGTTCATGTTTGTTTATTTCCGAATGCAACAAGCTCCGCATTACACCCGAACATC ACTCCAGATGAGGGCTTTCTGAGTGTGGGGTCAAATAGTTTCATGTTCCCCAAATGGCCCAAAACTGACAGTTTAAACGCTG TCTTGGAACCTAATATGACAAAAGCGTGATCTCATCCAAGATGAACTAAGTTTGGTTCGTTGAAATGCTAACGGCCAGTTGG TCAAAAAGAAACTTCCAAAAGTCGGCATACCGTTTGTCTTGTTTGGTATTGATTGACGAATGCTCAAAATAATCTCATTAAT GCTTAGCGCAGTCTCTCTATCGCTTCTGAACCCCGGTGCACCTGTGCCGAAACGCAAATGGGGAAACACCCGCTTTTTGGAT GATTATGCATTGTCTCCACATTGTATGCTTCCAAGATTCTGGTGGGAATACTGCTGATAGCCTAACGTTCATGATCAAAATTT AACTGTTCTAACCCCTACTTGACAGCAATATATAAACAGAAGGAAGCTGCCCTGTCTTAAACCTTTTTTTTTATCATCATTAT TAGCTTACTTTCATAATTGCGACTGGTTCCAATTGACAAGCTTTTGATTTTAACGACTTTTAACGACAACTTGAGAAGATCA AAAAACAACTAATTATTCGAAACATCATACAAAATG

\section{PPPALD4}

AACGGACAATAAGAAGAAATAAAAAGAAAAGCGGTGGGGGAGGGATTATTAAATAAGGATTATGTAACCCCAGGGTACCG TTCTATACATATTTAAGGATTATTTAGGACAATCGATGAAATCGGCATCAAACTGGATGGGAGTATAGTGTCCGGATAATCG GATAAATCATCTTGCGAGGAGCCGCTTGGTTGGTTGGTGAGAGGAGTGAAATATGTGTCTCCTCACCCAAGAATCGCGATA TCAGCACCCTGTGGGGGACACTATTGGCCTCCCTCCCAAACCTTCGATGTGGTAGTGCTTTATTATATTGATTACATTGATTA CATAGCTAAACCCTGCCTGGTTGCAAGTTGAGCTCCGAATTCCAATATTAGTAAAATGCCTGCAAGATAACCTCGGTATGGC GTCCGACCCCGCTTAATTATTTTAACTCCTTTCCAACGAGGACTTCGTAATTTTTGATTAGGGAGTTGAGAAACGGGGGGTC TTGATACCTCCTCGATTTCAGATCCCACCCCCTCTCAGTCCCAAGTGGGACCCCCCTCGGCCGTGAAATGCGCGCACTTTAG TTTTTTTCGCATGTAAACGCCGGTGTCCGTCAATTAAAAGTCGCAGACTAGGGTGAACTTTACCATTTTTGTCGCACTCCGTC TCCTCGGAATAGGGGTGTAGTAATTCTGCAGTAGTGCAATTTTTACCCCGCCAAGGGGGGGCGAAAAGAGACGACCTCATC ACGCATTCTCCAGTCGCTCTCTACGCCTACAGCACCGACGTAGTTAACTTTCTCCCATATATAAAGCAATTGCCATTCCCCTG AAAACTTTAACCTCTGCTTTTTCTTGATTTTTCCTTGCCCAAAGAAAAGATCATACAAAATG

\section{$\boldsymbol{P}_{\text {HpDHAS }}$}

AACGCTCGAGAGCCGTGGAACAGAGTCCCCTGAATCTCTTGCCAAGCGGCTTGCTGCTGCATCTGCGGAGATGGAGTACGC CAGGGCAGTGGACACGACAAGGTCATTGTCAACGATGACCTTGAGAAGGCGTACTCTGAGCTGAAGGAGTTCATTTTCGCC GAGCCCATCTAAGCATTCATAAATTTTTAATATCTAGAGCTCTCATACGGGACAGTATCTCCTCCAACCTTGCGTCAAGCTT GTCCTCTTCATGCTCCTCAACAGTCATGGCATCCAGCTGCTGCTGCTTTTGCTCCAGCCTGGCATATATGTCGCCATACAGCT TGAGTTGGATTTTGATGAAACTCTCAAAGGTAGGGTCCACCAGTGACAGTCGCAGCGCAATGAACTGCTCGATTTCGTTCTT GAGCCGTGTGTTGATGTCCGTGTAGATATTTTCTGCCTCGTCGTACTCAACTTTGAACTTCTGCAGCTTGTCCAGGCTCTTCT GTAACTGGTCTGTTTTCTCGGTGTGATGCTGCTCGGTCACCTGTCGCTCAATCGCTTCGTACTCGCTCTGCAGCTTCGAAACC TTGAATCGTGAAACGTCGTAATCCACCTTTTTGCGTGCGCGCTTCTTGATCAGCTTGTTGATCTCGTCGTTGTACTTCTTCAG CTCGTTAATCGGCTCCACGACCGTGATGCTCATTGGCTCCAGAATTTCTGGCAGAATATTGTCTTTGATGTCTACCACCATCT GCAGATAATTCAGAGAAATACCATCTCTGGGGTTCACCTTGTGCTCTTCTGGCCGTTCCGCAGCTTCCGACCGCTTATCAGC CTTGAGCTCAAAGCTATAGTCTCCGTAAAACGAGTCCAGTGTTCTAGCCATATTTATCTGAGTCTCGAGCAGATTCTCCGAA ATTGCCCACAAAACGGCCTAGTTCCTGGTCCAGCTCGTTGGTGTAAGTCTCGAGTTTGCGGAAATTGGCCTCCTGGACGTCA AACTCAGGATCAACAGAGGGCTCACCTTTGTTTGTGCGTAGTATCACATGTGCTCCGGCACGATTGACAGCTTTTTTAAACC CAACCCATGACATGTCGAGGAAAGGGTCGTTTCGGGGAGTTAAATATTTTTGGCTATGTAGCAGACATGTTTCGACGCTGGC GTCGCGTCGATCGGAAAATATTACCCCAGGAACAAGCACTTGCTTGGGTTAGCCACCACCCTGCGCAAGCCTTTTTGCCGGC TCTACACAGGGCCAATGAAATCTGGGCGGAATCTGAAACCGATGAAACGGACGACACTGGCAACAAGCTCACTGCACTATT TTATTTTTCTAGTGAAATAGCCTATCCTCGTCTCGCTCCCCTCATACCTGTAAAGGGGTGCAATTTAGCCTCGTTCCAGCCAT TCACGGGCCACTCAACAACACGTCGGCTACCATGGGGTGCTTGGGCACCAAAAGGCCTATAAATAGGCCCCCATCCGTCTG CTACACAGTCATCTCTGTCTTTTCTTCCCATCATACAAAATG

\section{$P_{\text {HPFMD }}$}

AACGAATGTATCTAAACGCAAACTCCGAGCTGGAAAAATGTTACCGGCGATGCGCGGACAATTTAGAGGCGGCGAATCAA GAAACACCTGCTGGGCGAGCAGTCTGGAGCACAGTCATCGATGGGCCCGAGATCCCACCGCGTTCCTGGGTACCGGGACGT GAGGCAGCGCGACATCCATCAAATATACCAGGCGCCAACCGAGTCTCTCGGAAAACAGCTTCTGGATATCTTCCGCTGGCG GCGCAACGACGAATAATAGTCCCTGGAGGTGACGGAATATATATGTGTGGAGGGTAAATCTGACAGGGTGTAGCAAAGGT AATATTTTCCTAAAACATGCAATCGGCTGCCCCGCGACGGGAAAAAGAATGACTTTGGCACTCTTCACCAGAGTGGGGTGT CCCGCTCGTGTGTGCAAATAGGCTCCCACTGGTCACCCCGGATTTTGCAGAAAAATAGCAAGTTCCGGGGTGTCTCACTGGT GTCCGCCAATAAGAGGAGCCGGCAGGCACGGAGTCTACATCAAGCTGTCTCCGATACACTCGACTACCATCCGGGTCTGTC AGAGGGGGGAATGGCACTATAAATACCGCCTCCTTGCGCTCTCTGCCTTCATCAATCAAATCATCATACAAAATG 
Supplemental Table 3 List and sequence information of utilized signal peptides

\begin{tabular}{|c|c|c|c|}
\hline Naturally secreted protein & Species & Abbreviation & Sequence \\
\hline $\begin{array}{l}\text { Mating pheromone al- } \\
\text { phafactor }\end{array}$ & $\begin{array}{l}\text { Saccharomyces cere- } \\
\text { visiae }\end{array}$ & Sce-Prepro & $\begin{array}{l}\text { MRFPSIFTAVLFAASSALAAPVNTTTEDETAQIPAEAVIGYLD } \\
\text { LEGDFDVAVLPFSNSTNNGLLFINTTIASIAAKEEGVSLDKREA }\end{array}$ \\
\hline Inulinase & $\begin{array}{l}\text { Kluyveromyces marx- } \\
\text { ianus }\end{array}$ & $K m a-$ Inulinase & MKLAYSLLLPLAGVSAA \\
\hline Invertase 2 & $\begin{array}{l}\text { Saccharomyces cere- } \\
\text { visiae }\end{array}$ & Sce-Invertase2 & MLLQAFLFLLAGFAAKISA \\
\hline Acid Phosphatase 5 & $\begin{array}{l}\text { Saccharomyces cere- } \\
\text { visiae }\end{array}$ & Sce-Acid Phosphatase & MFKSVVYSILAASLANAA \\
\hline GmaUPO & Galerina marginata & Gma-UPO & MRGTPIFASLIALFAHAAIAFPAYGSLAGLTREQLDEILPTLEIRA \\
\hline Serum Albumin & Homo sapiens & $H s a-$ Serum Albumin & MKWVTFISLLFLFSSAYSA \\
\hline Glucoamylase & Aspergillus awamori & Aaw-Glucoamylase & MSFRSLLALSGLVCSGLA \\
\hline Killer Protein K1 Toxin & $\begin{array}{l}\text { Saccharomyces cere- } \\
\text { visiae }\end{array}$ & Sce-Killer Protein & MTKPTQVLVRSVSILFFITLLHLVVA \\
\hline MroUPO & Marasmius rotula & Mro-UPO & MKLAISSSLIALVSVTTALANSQDVVDFGA \\
\hline CfoCPO & $\begin{array}{l}\text { Caldariomyces fum- } \\
\text { ago }\end{array}$ & $C f_{0}-\mathrm{CPO}$ & MFSKVLPFVGAVAALPHSVRA \\
\hline CglUPO & $\begin{array}{l}\text { Chaetomium glo- } \\
\text { bosum }\end{array}$ & $C g l-\mathrm{UPO}$ & MRTSLLPALAAVSPVLA \\
\hline CciUPO & Coprinopsis cinerea & $C c i-\mathrm{UPO}$ & $\begin{array}{l}\text { MISTSKHLFVLLPLFLVSHLSLVLGFPAYASLGGLTERQVEEYTS } \\
\text { KLPIVA }\end{array}$ \\
\hline$\alpha$ Amylase & Aspergillus niger & Ani- $\alpha$ Amylase & MVAWWSLFLYGLQVAAPALA \\
\hline$\alpha$ Galactosidase & $\begin{array}{l}\text { Saccharomyces cere- } \\
\text { visiae }\end{array}$ & Sce $-\alpha$ Galactosidase & MFAFYFLTACISLKGVFGA \\
\hline Lysozyme g2 & Gallus gallus & Gga-Lysozym & MLGKNDPMCLVLVLLGLTALLGICQGA \\
\hline $\begin{array}{l}\text { AaeUPO* }{ }^{*} \quad \text { (variant } \\
\text { PaDa-I) }\end{array}$ & Agrocybe aegerita & Aae-UPO* & MKYFPLFPTLVYAVGVVAFPDYASLAGLSQQELDAIIPTLEARA \\
\hline MthUPO & $\begin{array}{l}\text { Myceliophthora ther- } \\
\text { mophila }\end{array}$ & $M t h-\mathrm{UPO}$ & MRASVLPVLIAISPALA \\
\hline
\end{tabular}


Supplemental Table 4 Background information on utilized signal peptides and promoters

\begin{tabular}{|c|c|c|c|c|c|}
\hline Signal Peptide & Reference for use in yeast & Promoter & Naturally regulated protein & $\begin{array}{l}\text { Promoter } \\
\text { strength }\end{array}$ & Reference \\
\hline Sce-Prepro & Brake et al. $1984^{7}$ & $P_{P p P M P 20}$ & $\begin{array}{l}\text { peroxisomal glutathione } \\
\text { peroxidase }\end{array}$ & Strong & Vogl et al. $2016^{8}$ \\
\hline Kma-Inulinase & Chung et al. $1996^{9}$ & $P_{\text {HpMOX }}$ & methanol oxidase & Strong & Vogl et al. $2020^{10}$ \\
\hline Sce-Invertase2 & Chang et al. $1986^{11}$ & $P_{P p F L D 1}$ & $\begin{array}{l}\text { formaldehyde dehydro- } \\
\text { genase }\end{array}$ & Strong & Vogl et al. $2016^{8}$ \\
\hline Sce-Acid Phosphatase & Sidhu et al. $1991^{12}$ & $P_{P p F D H 1}$ & formate dehydrogenase & Strong & Vogl et al. $2016^{8}$ \\
\hline Gma-UPO & Püllmann et al. $2020^{1}$ & $P_{P p D A S I}$ & $\begin{array}{l}\text { dihydroxyacetone syn- } \\
\text { thase } 1\end{array}$ & Strong & Vogl et al. $2016^{8}$ \\
\hline$H s a-$ Serum Albumin & Xiong et al. $2010^{13}$ & $P_{P p D A S 2}$ & $\begin{array}{l}\text { dihydroxyacetone syn- } \\
\text { thase } 2\end{array}$ & Strong & Vogl et al. $2016^{8}$ \\
\hline Aaw-Glucoamylase & Fierobe et al. $1997^{14}$ & $P_{P p C A T I}$ & catalase 1 & Strong & Vogl et al. $2016^{8}$ \\
\hline Sce-Killer Protein & Tokunaga et al. $1988^{15}$ & $P_{P p A O X I}$ & alcohol oxidase 1 & Strong & Vogl et al. $2016^{8}$ \\
\hline Mro-UPO & Püllmann et al. $2020^{1}$ & $P_{P p A L D 4}$ & $\begin{array}{l}\text { mitochondrial aldehyde } \\
\text { dehydrogenase }\end{array}$ & Weak & $\begin{array}{l}\text { Prielhofer et al. } \\
2017^{4}\end{array}$ \\
\hline$C f_{o}-\mathrm{CPO}$ & Püllmann et al. $2020^{1}$ & $P_{\text {HpDHAS }}$ & $\begin{array}{l}\text { dihydroxyacetone syn- } \\
\text { thase }\end{array}$ & Strong & Saraya et al. $2012^{16}$ \\
\hline$C g l-\mathrm{UPO}$ & Püllmann et al. $2020^{1}$ & $P_{H p F M D}$ & formate dehydrogenase & Strong & Vogl et al. $2020^{10}$ \\
\hline $\mathrm{Cci}-\mathrm{UPO}$ & Püllmann et al. $2020^{1}$ & & & & \\
\hline Ani- $\alpha$ Amylase & Kapat et al. $1998^{17}$ & & & & \\
\hline Sce $-\alpha$ Galactosidase & $\begin{array}{l}\text { Hofmann and Schultz } \\
1991^{18}\end{array}$ & & & & \\
\hline Gga-Lysozym & Tsuchiya et al. $1993^{19}$ & & & & \\
\hline Aae-UPO* & $\begin{array}{l}\text { Molina-Espeja et al. } \\
2014^{20}\end{array}$ & & & & \\
\hline Mth-UPO & Püllmann et al. $2020^{1}$ & & & & \\
\hline
\end{tabular}


Supplemental Table 5 Protein sequences of the utilized UPOs and other enzymes

A/S- Connecting amino acid for Golden Gate assembly

\section{MhiUPO}

UPO derived from Myceliophthora hinnulea

AGFDTWSPPGPTDVRAPCPMLNTLANHGFLPHDGKDITREQTENALFDALNINKTLASFLFDFALTTNPKNTSTFSLNDLGNHNIL EHDASLSRADAYFGNVLQFNQTVFDETKTYWEGDTIDLRMAAKARLGRIKTSQATNPTYSMSELGDAFTYGESAAYVVVLGDL ESRTVNRSWVEWFFEHEQLPQHLGWKRPAVSFEEEDLNRFMEEIEKYTKGLEGSNSTSGSQKHRRRLPRRRTHFGFS

\section{MfeUPO}

UPO derived from Myceliophthora fergusii

AGFDTWSPPGPYDVRAPCPMLNTLANHGFLPHDGKDITRENTENALFEALHINKTLGSFLFDFALTTNPRNTSTFSLNDLGNHNIL EHDASLSRADAYFGNVLQFNQTVFDETKTYWDGDVIDLRMAARARLGRIKTSQATNPTYSMSELGDAFTYGESAAYVVVLGDK ESRTAKRSWVEWFFEHEQLPQHLGWKRPASSLEEEDLFTIMDEIRQYTSELEGSTSSSDAQTSRRQLPRRRTHFGFS

\section{DcaUPO}

UPO derived from Daldinia caldariorum

AGFAPWKAPGPDDVRGPCPMLNTLANHGFLPHDGKNIDVNTTVNALSSALNLDDELSRDLHTFAVTTNPQPNATWFSLNHLSRH NVLEHDASLSRQDAYFGPPDVFNAAVFNETKAYWTGDIINFQMAANALTARLMTSNLTNPEFSMSQLGRGFGLGETVAYVTILG SKETRTVPKAFVEYLFENERLPYELGFKKMKSALTEDELTTMMGEIYSLQHLPESFTKPFAKRSEAPFEKRAEKRCPFHS

\section{CalB}

lipase B derived from Candida antarctica

ALPSGSDPAFSQPKSVLDAGLTCQGASPSSVSKPILLVPGTGTTGPQSFDSNWIPLSTQLGYTPCWISPPPFMLNDTQVNTEYMVN AITALYAGSGNNKLPVLTWSQGGLVAQWGLTFFPSIRSKVDRLMAFAPDYKGTVLAGPLDALAVSAPSVWQQTTGSALTTALR NAGGLTQIVPTTNLYSATDEIVQPQVSNSPLDSSYLFNGKNVQAQAVCGPLFVIDHAGSLTSQFSYVVGRSALRSTTGQARSADY GITDCNPLPANDLTPEQKVAAAALLAPAAAAIVAGPKQNCEPDLMPYARPFAVGKRTCSGIVTPS

\section{Mrl2}

laccase derived from Moniliophthora roreri

ASIGPIADLVISNQDVSPDGFTRSAVVAGGDTIGPLIVGNKNDNLQINVVNNLDDDTMLQSTSIHWHGFFQQSTNWADGTAFVNQ CPIAKGNSFLYDFDATDQAGTFWYHSHLSTQYCDGLRGPIVIYDPDDPHASLYDVDDESTVITLADWYHTKAKEITFGTPDSTLIN GLGRWSQGNETDLSVITVTSGQRYRMRLINTACDAAYTFSIDNHTMTVIEADAVNIEPIEVDSLTIYAGQRYSFVLNADQAVGNY WIRANPNIGTMGYTNGINSAILRYDTAEEEEPDVLDITSTNSLSEADLVPLENPGAPGDPVVGGVDYALHLDFAFTSAATFTVNDA TFVPPTVPVLLQILSGAQTADTLLPSGSVVALPSNSTIELSMTGGLLGLEHPIHLHGHNFDVVRVAGSTEYNYENPIRRDVVNAGS TSDNVTIRFTTDNPGPWILHCHIDWHLEAGFAIVFAEATDEWVDTIDPSDAWENLCPTYDALSDDDLGS 


\section{Supplemental Table 6 Distribution of promoters and signal peptides amongst the sequenced hits}

Overview of the occurrence and distribution of respective promoter and signal peptides parts among the sequenced top hits (80 in total) for the eight tested enzymes. Redundant combinations have not been included.

\section{Promoter}

\begin{tabular}{|l|l|l|}
\hline Promoter & Occurrence & Percentage \\
\hline$P_{P p P M P 20}$ & 3 & $3.8 \%$ \\
\hline$P_{H p M O X}$ & 4 & $5 \%$ \\
\hline$P_{P p F L D 1}$ & 19 & $23.8 \%$ \\
\hline$P_{P p F D H 1}$ & 11 & $13.8 \%$ \\
\hline$P_{P p D A S 1}$ & 1 & $1.3 \%$ \\
\hline$P_{P p D A S 2}$ & 1 & $1.3 \%$ \\
\hline$P_{P p C A T 1}$ & 9 & $11.3 \%$ \\
\hline$P_{P p A O X 1}$ & 2 & $2.5 \%$ \\
\hline$P_{P p A L D 4}$ & 12 & $15 \%$ \\
\hline$P_{H p D H A S}$ & 5 & $6.3 \%$ \\
\hline$P_{H p F M D}$ & 13 & $16.3 \%$ \\
\hline
\end{tabular}

\section{Signal Peptides}

\begin{tabular}{|l|l|l|}
\hline Signal Peptide & Occurrence & Percentage \\
\hline Sce-Prepro & 3 & $3.8 \%$ \\
\hline Kma-Inulinase & 4 & $5 \%$ \\
\hline Sce-Invertase 2 & 6 & $7.5 \%$ \\
\hline Sce-Acid Phosphatase & 1 & $1.3 \%$ \\
\hline Gma-UPO & 9 & $11.3 \%$ \\
\hline Hsa-Serum Albumin & 3 & $3.8 \%$ \\
\hline Aaw-Glucoamylase & 5 & $6.3 \%$ \\
\hline Sce-Killer Protein & 2 & $2.5 \%$ \\
\hline$M r o-$ UPO & 9 & $11.3 \%$ \\
\hline$C f o-$ CPO & 2 & $2.5 \%$ \\
\hline$C g l-$ UPO & 1 & $1.3 \%$ \\
\hline$C c i-$ UPO & 0 & $0 \%$ \\
\hline Ani- $\alpha$ Amylase & 8 & $10 \%$ \\
\hline Sce- $\alpha$ Galactosidase & 10 & $12.5 \%$ \\
\hline Gga-Lysozym & 10 & $12.5 \%$ \\
\hline Aae-UPO* & 2 & $2.5 \%$ \\
\hline Mth-UPO & 5 & $6.3 \%$ \\
\hline
\end{tabular}




\section{Supplemental Table 7 Numeric summary of TteUPO error prone PCR libraries}

Screening as described in Supplemental Figure 16. Display of calculated threshold values of the obtained libraries.

\begin{tabular}{|c|c|c|c|c|}
\hline $\begin{array}{l}\mathrm{MnCl}_{2} \text { concentra- } \\
\text { tion }\end{array}$ & above $50 \%$ activity treshold & above $140 \%$ activity treshold & inactive $(<10 \%)$ activity treshold & highest mean activity \\
\hline 0.01 & $54 \%$ & $1 \%$ & $27 \%$ & $144 \%$ \\
\hline 0.05 & $54 \%$ & $1 \%$ & $33 \%$ & $297 \%$ \\
\hline 0.1 & $26 \%$ & none & $51 \%$ & $129 \%$ \\
\hline 0.2 & $21 \%$ & $1 \%$ & $60 \%$ & $171 \%$ \\
\hline 0.5 & $7 \%$ & $0.5 \%$ & $81 \%$ & $220 \%$ \\
\hline
\end{tabular}

\section{References}

1. Püllmann, P.; Knorrscheidt, A.; Münch, J.; Palme, P. R.; Hoehenwarter, W.; Marillonnet, S.; Alcalde, M.; Westermann, B.; Weissenborn, M. J., A modular two yeast species secretion system for the production and preparative application of fungal peroxygenases. bioRxiv 2020, 2020.07.22.216432.

2. Liachko, I.; Dunham, M. J., An autonomously replicating sequence for use in a wide range of budding yeasts. FEMS Yeast Res. 2014, 14 (2), 364-367.

3. Camattari, A.; Goh, A.; Yip, L. Y.; Tan, A. H. M.; Ng, S. W.; Tran, A.; Liu, G.; Liachko, I.; Dunham, M. J.; Rancati, G., Characterization of a panARS-based episomal vector in the methylotrophic yeast Pichia pastoris for recombinant protein production and synthetic biology applications. Microb. Cell Fact. 2016, 15 (1), 139.

4. $\quad$ Prielhofer, R.; Barrero, J. J.; Steuer, S.; Gassler, T.; Zahrl, R.; Baumann, K.; Sauer, M.; Mattanovich, D.; Gasser, B.; Marx, H., GoldenPiCS: a Golden Gate-derived modular cloning system for applied synthetic biology in the yeast Pichia pastoris. BMC Syst. Biol. 2017, 11 (1), 123.

5. $\quad$ Lin-Cereghino, J.; Wong, W. W.; Xiong, S.; Giang, W.; Luong, L. T.; Vu, J.; Johnson, S. D.; Lin-Cereghino, G. P., Condensed protocol for competent cell preparation and transformation of the methylotrophic yeast Pichia pastoris. BioTechniques 2005, 38 (1), 44-48.

6. Faber, K. N.; Haima, P.; Harder, W.; Veenhuis, M.; Ab, G., Highly-efficient electrotransformation of the yeast Hansenula polymorpha. Current Genetics 1994, 25 (4), 305-310.

7. Brake, A. J.; Merryweather, J. P.; Coit, D. G.; Heberlein, U. A.; Masiarz, F. R.; Mullenbach, G. T.; Urdea, M. S.; Valenzuela, P.; Barr, P. J., Alpha-factor-directed synthesis and secretion of mature foreign proteins in Saccharomyces cerevisiae. PNAS 1984, 81 (15), $4642-4646$.

8. Vogl, T.; Sturmberger, L.; Kickenweiz, T.; Wasmayer, R.; Schmid, C.; Hatzl, A.-M.; Gerstmann, M. A.; Pitzer, J.; Wagner, M.; Thallinger, G. G.; Geier, M.; Glieder, A., A Toolbox of Diverse Promoters Related to Methanol Utilization: Functionally Verified Parts for Heterologous Pathway Expression in Pichia pastoris. ACS Synth. Biol. 2016, 5 (2), 172-186.

9. Chung, B. H.; Nam, S. W.; Kim, B. M.; Park, Y. H., Highly efficient secretion of heterologous proteins from Saccharomyces cerevisiae using inulinase signal peptides. Biotechnol. Bioeng. 1996, 49 (4), 473-479.

10. Vogl, T.; Fischer, J. E.; Hyden, P.; Wasmayer, R.; Sturmberger, L.; Glieder, A., Orthologous promoters from related methylotrophic yeasts surpass expression of endogenous promoters of Pichia pastoris. AMB Expr. 2020, 10 (1), 38.

11. Chang, C. N.; Matteucci, M.; Perry, L. J.; Wulf, J. J.; Chen, C. Y.; Hitzeman, R. A., Saccharomyces cerevisiae secretes and correctly processes human interferon hybrid proteins containing yeast invertase signal peptides. Molecular and cellular biology 1986, 6 (5), $1812-1819$.

12. Sidhu, R. S.; Mathewes, S.; Bollon, A. P., Selection of secretory protein-encoding genes by fusion with PHO5 in Saccharomyces cerevisiae. Gene 1991, 107 (1), 111-118.

13. Xiong, R.; Chen, J.; Chen, J., Secreted expression of human lysozyme in the yeast Pichia pastoris under the direction of the signal peptide from human serum albumin. Biotechnology and Applied Biochemistry 2008, 51 (3), 129-134.

14. Fierobe, H.-P.; Mirgorodskaya, E.; Frandsen, T. P.; Roepstorff, P.; Svensson, B., Overexpression and Characterization of Aspergillus awamoriWild-Type and Mutant Glucoamylase Secreted by the Methylotrophic YeastPichia pastoris:Comparison with Wild-Type Recombinant Glucoamylase Produced UsingSaccharomyces cerevisiaeandAspergillus nigeras Hosts. Protein Expr. Purif. 1997, 9 (2), 159-170.

15. Tokunaga, M.; Wada, N.; Hishinuma, F., A novel yeast secretion signal isolated from $28 \mathrm{~K}$ killer precursor protein encoded on the linear DNA plasmid pGKL1. Nucleic acids research 1988, 16 (15), 7499-7511.

16. Saraya, R.; Krikken, A. M.; Kiel, J. A. K. W.; Baerends, R. J. S.; Veenhuis, M.; van der Klei, I. J., Novel genetic tools for Hansenula polymorpha. FEMS Yeast Res. 2012, 12 (3), 271-278.

17. Kapat, A.; Jung, J.-K.; Park, Y.-H., Enhancement of extracellular glucose oxidase production in pH-stat feed-back controlled fed-batch culture of recombinant Saccharomyces cerevisiae. Biotechnol. Lett. 1998, 20 (7), 683-686.

18. Hofmann, K. J.; Schultz, L. D., Mutations of the $\alpha$-galactosidase signal peptide which greatly enhance secretion of heterologous proteins by yeast. Gene 1991, 101 (1), 105-111.

19. Tsuchiya, Y.; Fujisawa, H.; Nakayama, K.-I.; Nagahora, H.; Jigami, Y., Effect of chicken lysozyme signal peptide alterations on secretion of human lysozyme in Saccharomyces cerevisiae. Biochemistry and Cell Biology 1993, 71 (7-8), 401-405.

20. Molina-Espeja, P.; Garcia-Ruiz, E.; Gonzalez-Perez, D.; Ullrich, R.; Hofrichter, M.; Alcalde, M., Directed evolution of unspecific peroxygenase from Agrocybe aegerita. Appl. Environ. Microbiol. 2014, 80 (11), 3496-507. 